\title{
A CERTAIN CLASS OF TOPOLOGICAL PROPERTIES ${ }^{1}$
}

\author{
R. L. WILDER ${ }^{2}$
}

I. Introduction. About thirty years ago it was considered that Topology had two aspects, according to which its practitioners could be roughly divided into two schools. These aspects would today be termed global and local, and those whose interests lay mainly in global properties of a space or configuration formed one school, while those whose chief interest lay in local properties formed the other. The causes for this division of interests were methodological: The combinatorial method, stemming chiefly from the work of Poincaré and Veblen, yielded only global invariants such as the classical Betti numbers and duality theorems; while the set-theoretic method, starting from the strictly local notion of limit point, was particularly suited for the study of local properties. Thus a more accurate differentiation between the schools could be made according to method; for while the set-theoretic method allowed of the study of such concepts as local connectedness, the concepts of connectedness and unicoherence, for example, were global; and soon the combinatorial method became adapted to the study of local properties. ${ }^{3}$

While this division into schools has essentially disappeared (although it is still convenient to distinguish the set-theoretic and algebraic methods), the distinction between global and local aspects of topological spaces furnishes a useful theoretical perspective. It is the purpose of the present discussion to focus attention on properties which in general lie between the global and local, and which might therefore be termed medial. ${ }^{4}$ For example, the property of every open subset of a space being a $G_{\delta}$ is medial, in that it holds for all open sets, large and small.

As in the case of such local properties as that of local connectedness, however, we find it more fruitful, in defining medial properties, to use pairs of open sets rather than single open sets. The original definition of local connectedness at a point $x$ stipulated that for arbitrary open set $U$ containing $x$ there exists an open set $V$ such that

1 Presidential Address delivered before the Annual Meeting of the Society in Cincinnati on January 29, 1958; received by the editors September 4, 1959.

${ }^{2}$ Aid in the preparation of this address was received from National Science Foundation Grants G-2783 and G-5612.

${ }^{3}$ For a more extended discussion of the situation thirty years ago, see my symposium lecture [9].

${ }^{4}$ We resist the temptation to introduce the barbarism "glocal," on grounds of its ease of confusion with "global." 
$x \in V \subset U$ and $V$ lies in one component of $U$. And although in the case where a space is locally connected at every point the above definition is equivalent to requiring merely that every point have arbitrarily small connected neighborhoods, it turns out that for the so-called $r$-dimensional local connectedness, $r>0$, it is necessary to go back to the double neighborhood type of definition in order to obtain a topologically fruitful notion. Moreover, it would, for instance, yield only triviality to turn connectedness into a medial property of a space $X$ by stipulating that all open subsets of the space shall be connected; $X$ would immediately be seen to be of necessity a degenerate space! (We assume Hausdorff spaces throughout.) Or, if we stipulated that each open set have only a finite number of components, then the space would of necessity be finite. On the other hand, if we require for every pair of open sets $P$ and $Q$, $P \supset \bar{Q}$ and $\bar{Q}$ compact, that $Q$ lie in a finite number of components of $P$, we get a significant property; if $X$ is locally compact, it will then be locally connected at every point.

There is a type of medial property which I have found very fruitful, but whose significance I did not realize even though I had used it in a number of different situations. Let us call a pair of open sets $P$ and $Q$ a canonical pair if $P \supset \bar{Q}$ and $\bar{Q}$ is compact. Let $G(U)$ be a group which is a function of the open sets $U$ of a given space $X$, such that for every canonical pair $P, Q$, there exists a homomorphism

$$
i_{*}: G(Q) \rightarrow G(P)
$$

induced by the inclusion mapping $i: Q \rightarrow P$. For example, $G(U)$ might be the homology group generated by compact cycles and homologies in $U$. In particular, if $G(U)$ is the 0-dimensional homology group over a field, and $i_{*} G(\bar{Q})$ is required always to be finite dimensional in every homomorphism of type (0), then, again assuming $X$ to be locally compact Hausdorff, we get a property which is equivalent to that of the local connectedness of $X$. If $G(U)$ is the $r$-dimensional homology group, $r>0$, then a more significant property is obtained, much stronger than the local $r$-connectedness of the space, by requiring that $i_{*} G(Q)$ always be finitely generated. To summarize: The type of medial property we have in mind is based upon the fixing of a particular kind of group $G(U)$, together with the stipulation that for every canonical pair $P, Q$, the image $i_{*} G(Q)$ in $(0)$ is finitely generated.

It will be found that a number of theorems which were originally stated in terms of local properties find significant generalizations in terms of medial properties. 
Throughout, $X$ will denote a locally compact Hausdorff space, unless otherwise specified. Open subsets of $X$ will be denoted by $U, V, W, P, Q, R$ with appropriate indices as required. Cech homology and cohomology with a field as coefficient domain are used throughout. For $M$ a compact set, $H_{r}(M)$ and $H^{r}(M)$ denote the homology and cohomology groups of dimension $r$, respectively. And if $M$ is not compact, then $H$ may be replaced either by $h$ to denote compact supports in both homology and cohomology, or by $\mathfrak{S}$ to denote corresponding groups "mod infinity"; for instance, $\mathfrak{S}_{r}(M)$ denotes the homology group $H_{r}(\hat{M}, \hat{p})$, where $\hat{M}$ denotes $M$ compactified by addition of the ideal point $\hat{p}$. Finally, whatever the group $G(U)$, the image of $i_{*}$ in (0) may be denoted by $G(Q \mid P)$.

The principal medial properties with which we shall be concerned are the following:

Definition I.1. Property $(P, Q)_{r}$. A subset $M$ of $X$ has property $(P, Q)_{r}$ if for every canonical pair $P, Q$ of open subsets of $X$, the group $h_{r}(M \cap Q \mid M \cap P)$ is finitely generated.

REMARK. While in case $M=X$, property $(P, Q)_{r}$ is a topological property of $X$, for a subset $M \subset X$, it is a positional property. More precisely, so long as only canonical pairs $P, Q$ of the space $M$ are employed, the property remains invariant; but if $M$ is imbedded in a larger space $X$, then possession of the property by $M$ depends upon its position in $X$. The following example will make this clearer:

EXAMPLE I.1. In the cartesian plane $E^{2}$, let $A=\{(x, y) \mid 0<x$ $\leqq 1 / \pi, y=\sin (1 / x)\}$ and $B=\{(x, y) \mid x=0,-1 \leqq y \leqq 1\}$; let $C$ be an arc joining $(1 / \pi, 0)$ and $(0,-1)$ in the fourth quadrant of $E^{2}$ but not meeting $A \cup B$ otherwise. If $M$ is the bounded domain whose boundary is $A \cup B \cup C$, then $M$ does not have property $(P, Q)_{0}$. This is evident if $P$ and $Q$ are circles with centers at $(0,0)$ and radii $1 / 2$ and $1 / 4$ respectively. However, $M$ is homeomorphic with the open circular disk bounded by $x^{2}+y^{2}=1$ which does have property $(P, Q)_{0}$.

By way of contrast, the set $X=A \cup B$ does not have property $(P, Q)_{0}$, and this will be a topological invariant of $X$ (because of the compactness of $X$ and the consequent equivalence of its open subsets with the intersections by open subsets of any space in which it may be imbedded).

REMARK. In 1920, when topological characterizations of those spaces that are continuous images of the real number interval ("continuous curves") were being sought, Sierpinski [6] showed that a necessary and sufficient condition that a metric continuum $C$ be such an image is that for arbitrary positive number $\epsilon, C$ be the union of a 
finite number of continua $C_{i}$ of diameter $\langle\epsilon$; thus showing that the global property so defined is equivalent, for compacta, to the local connectedness previously shown (by Hahn and Mazurkiewicz) to characterize continuous curves. It is easy to show that Sierpinski's global property is equivalent, for compacta, to property $(P, Q)_{0}$. R. L. Moore later [5] named the Sierpinski property, with the restriction that the $C_{i}$ be compact deleted, "Property S," and exploited it in investigations of positional properties of locally connected sets in the plane, etc.

In [11], I studied a property which I called "Property $\mathrm{S}_{n}$," a generalization of the notion of "Property $S$ " to higher dimensions. In [13] this notion was generalized to one called "Property $S_{r}$ rel $G^{r}$," where $G^{r}$ was a special group of $r$-cycles, and in [13, p. 236, Theorem 7.9] equivalence with the corresponding " $P, Q$ " property shown; immediately after which a definition $[13$, p. 237, 7.12] of "Property $(P, Q)^{r}$ rel $G^{r}$ " was given. Previous to the latter, and independently thereof, in $\left[13\right.$, p. 193] I defined "Property $(P, Q)_{r}$ " for cohomology (the " $(P, Q)^{r}$ " defined below) and used it in the study of generalized manifolds. Association of the two properties was finally made in Chapter XI of [13], where in particular the "First fundamental duality theorem" (Theorem II.1 below) of the present paper was established for the compact case.

Definition I.2. Property $(P, Q)^{r}$. A subset $M$ of $X$ has property $(P, Q)^{r}$ if for every canonical pair $P, Q$ of open subsets of $X$, the group $h^{r}(M \cap Q \mid M \cap P)$ is finitely generated. Thus this property differs from $(P, Q)_{r}$ only in that (compact) cohomology replaces (compact) homology.

Definition I.3. Property $(P, Q, \sim)_{r}$. This differs from $(P, Q)_{r}$ only in that the group of bounding compact $r$-cycles of $M$ is involved. More precisely, a subset $M$ of $X$ has property $(P, Q)_{r}$ if for every canonical pair $P, Q$ of open subsets of $X$, the image of $i \partial$ in the sequence of homomorphisms

$$
h_{r+1}(M, M \cap Q) \stackrel{\partial}{\rightarrow} h_{r}(M \cap Q) \stackrel{i}{\rightarrow} h_{r}(M \cap P)
$$

where $i$ is induced by inclusion, is finitely generated.

An equivalent definition which we shall find useful is the following: In the sequence of homomorphisms

$$
h_{r+1}(M, M \cap Q) \stackrel{\partial}{\rightarrow} h_{r}(M \cap Q) \stackrel{i_{1}}{\rightarrow} h_{r}(M \cap P) \stackrel{i_{2}}{\rightarrow} h_{r}(M)
$$

where $i_{1}$ and $i_{2}$ are induced by inclusion, let $L$ denote the kernel of $i_{2} i_{1}$; then $i_{1} L$ is finitely generated. 
REMARK. Since we assume $X$ to be locally compact throughout, the set $P$ in any canonical pair may always be assumed to have a compact closure. Hence for $X$ to have property $(P, Q, \sim)_{r}$ it is necessary and sufficient that in the sequence of homomorphisms

$$
h_{r+1}(X, \bar{Q}) \stackrel{\partial}{\rightarrow} H_{r}(\bar{Q}) \stackrel{i}{\rightarrow} H_{r}(\bar{P})
$$

where $i$ is induced by inclusion, the group $i \partial h_{r+1}(X, \bar{Q})$ be finitely generated. Similarly, for $X$ to have property $(P, Q)_{r}$ it is necessary and sufficient that $H_{r}(\bar{Q} \mid \bar{P})$ be finitely generated for every canonical pair in which $\bar{P}$ is compact.

Referring to Example I.1, neither $A \cup B$ nor the domain $M$ has property $(P, Q, \sim)_{0}$.

Definition I.4. Property $(P, Q, \sim)^{r}$. This differs from $(P, Q, \sim)_{r}$ only in that cohomology is involved instead of homology. For example, for a locally compact space $X$ to have $(P, Q, \sim)^{r}$, it is required that for every canonical pair $P, Q$, the image of $i \delta$ obtained from the cohomology sequence

$$
h^{r-1}(X, Q) \stackrel{\delta}{\rightarrow} h^{r}(Q) \stackrel{i}{\rightarrow} h^{r}(P)
$$

be finitely generated.

REMARK. Clearly if a set $M$ has property $(P, Q)_{r}$, then $M$ has $(P, Q, \sim)_{r}$. However, the reverse does not necessarily hold. This is shown by the following example.

ExAmple I. 2. With $X=E^{3}$, let $M$ consist of a denumerable set of circles $C_{n}$, disjoint except that $C_{n}$ is tangent to $C_{n+1}, n=1,2,3, \cdots$, and converging to a point $p$ (a picture may be found in $[13$, p. 341]). Then both $M$ and $X-M$ have property $(P, Q, \sim)_{1}$, but neither has $(P, Q)_{1}$.

AbBreviations. Whenever we are dealing with a homomorphism $\phi: G \rightarrow H$, we may designate the image of $G$ in $H$ by $\operatorname{Im} \phi$. And we may designate the kernel of $\phi$ by $\operatorname{Kern} \phi$.

That a group $G$ is finitely generated may be expressed by writing " $G$ is f.g."

That a set $A$ contains the closure of a set $B$ may be denoted by the expression " $A \supset B$."

That a proof has been completed may be indicated by the sign at the end of the proof.

II. Duality theorems. We first prove certain relations between the various properties defined above, which we call "Fundamental Duality Theorems." 
TheOREM II.1 (First FUNDAMENTAL DUALITY THEOREM). In order that a locally compact space $X$ should have property $(P, Q, \sim)_{r-1}, r \geqq 1$, it is necessary and sufficient that $X$ have property $(P, Q, \sim)^{r}$.

(In [13], this theorem was given only for the case where $X$ is compact; see [13, p. 327, Theorem 3.3]. A simpler proof than that given below can be given for the compact case.)

ProOF OF NECEssity. As remarked above, we may assume, since $X$ is locally compact, that $\bar{P}$ is also compact. And we need only show that in the cohomology sequence (4), Im $i \delta$ is f.g. Now the dual of (4) is

$$
\mathfrak{S}_{r-1}(X-Q) \stackrel{\partial}{\leftarrow} H_{r}(X, X-Q) \stackrel{i_{1}}{\leftarrow} H_{r}(X, X-P) .
$$

Consider the diagram

$$
\mathfrak{S}_{r-1}(X-Q) \stackrel{\partial}{\leftarrow} H_{r}(\bar{P}, \bar{P}-Q) \stackrel{i_{1}}{\leftarrow} H_{r}(\bar{P}, \bar{P}-P)
$$

in which the $H_{r}(X, X-Q)$ and $H_{r}(X, X-P)$ of $\left(4^{\prime}\right)$ have been replaced by $H_{r}(\bar{P}, \bar{P}-Q)$ and $H_{r}(\bar{P}, \bar{P}-P)$ respectively. This diagram is commutative and $\operatorname{Im} i_{2} \partial$ is f.g. since $X$ has property $(P, Q, \sim)_{r-1}$. It follows that $\operatorname{Im} \partial i_{1}$ is f.g. and hence also that $\operatorname{Im} i \delta$ in (4) is f.g.

Proof of sufficiency. With a canonical pair $P, Q$ as in the proof above, let $P_{1}, Q_{1}$ be open sets such that $P \supset P_{1} \supset Q_{1} \supset Q$. It will be sufficient to show in the sequence of homomorphisms

$$
h_{r}(X, \bar{Q}) \stackrel{\partial}{\rightarrow} H_{r-1}(\bar{Q}) \stackrel{i}{\rightarrow} H_{r-1}\left(\bar{Q}_{1}\right),
$$

obtained from (3) by replacing $r+1$ by $r$ and $H_{r}(\bar{P})$ by $H_{r}\left(\bar{Q}_{1}\right)$, that $\operatorname{Im} i \partial$ is f.g. The dual of $\left(3^{\prime}\right)$ is

$$
\mathfrak{S}^{r}(X-\bar{Q}) \stackrel{\delta}{\leftarrow} H^{r-1}(X, X-\bar{Q}) \stackrel{i^{*}}{\leftarrow} H^{r-1}\left(X, X-\bar{Q}_{1}\right) .
$$

Consider the diagram

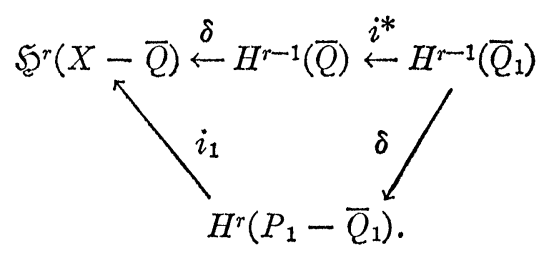


This is commutative, and $\operatorname{Im} i_{1} \delta$ is f.g. since $X$ has property $(P, Q, \sim)^{r}$. Consequently $\operatorname{Im} \delta i^{*}$ is f.g., and this implies that in $\left(3^{\prime}\right)$, Im $i \partial$ is f.g.

REMARK. While Theorem II.1 continues to be valid for closed subsets of a locally compact space (as a positional property), it does not generally hold for nonclosed subsets (not even for open subsets). For example, the set $M$ of Example I.1, as a subset of $E^{2}$, has property $(P, Q, \sim)_{1}$, but not property $(P, Q, \sim)^{2}$.

We recall that if $X$ is compact and $M$ a closed subset of $X$, and if $X$ is acyclic in dimensions $r$ and $r+1$, then the groups $H_{r}(M)$ and $H_{r+1}(X, M)$ are isomorphic (a direct consequence of the exactness of the homology sequence of the compact pair $X, M$; cf. [3, p. 11]). The second fundamental duality theorem will be an analogue of this. First, however, we need another definition and two preliminary theorems.

Definition II.1. If for every canonical pair of type $X, Q \operatorname{dim} h^{r}(Q \mid X)$ is finite, then we say $X$ has property $(X, Q)^{r}$. The corresponding homology property $(X, Q)_{r}$ is defined similarly.

REMARK. If we denote $\operatorname{dim} h^{r}(X)$ by $p^{r}(X)$, then we distinguish three cases: (1) $p^{r}(X)$ may be finite; (2) $p^{r}(X)$ may be infinite, yet $X$ have property $(X, Q)^{r}$, in which case we write $p^{r}(X)=\omega ;(3) p^{r}(X)$ may be infinite and $X$ fail to have $(X, Q)^{r}$, in which case we write $p^{r}(X)=\infty$. The expression $p^{r}(X) \leqq \omega$ denotes that case (3) is excluded, and evidently this is equivalent to $X$ having property $(X, Q)^{r}$.

Similar comments may be made for $p_{r}(X)=\operatorname{dim} h_{r}(X)$.

Theorem II.2a. Let $X$ have properties $(P, Q, \sim)^{r+1}$ and $(X, Q)^{r}$. If $M$ is a closed subset of $X$ having property $(P, Q, \sim)^{r}$, then $X-M$ has property $(P, Q, \sim)^{r+1}$.

Proof. Let $R, Q$ be a canonical pair and select an open set $P$ having compact closure such that $R \supset P \supset Q$. Consider the following diagram:

$$
\begin{aligned}
& h^{r}(X) \stackrel{j}{\rightarrow} h^{r}(M) \stackrel{\delta}{\longrightarrow} h^{r+1}(X-M) \\
& \begin{array}{cc}
\uparrow \phi_{3} & \uparrow g_{3} \\
h^{r}(R \cap M) \stackrel{\delta_{1}}{\rightarrow} h^{r+1}(R-M)
\end{array} \\
& \begin{array}{cc}
\uparrow \phi_{2} & \uparrow g_{2} \\
h^{r}(P \cap M) \stackrel{\delta_{2}}{\rightarrow} & h^{r+1}(P-M) \stackrel{i_{2}}{\rightarrow} h^{r+1}(P)
\end{array} \\
& \begin{array}{cr}
\uparrow g_{1} & \uparrow h_{1} \\
h^{r+1}(Q-M) \stackrel{i_{3}}{\rightarrow} & h^{r+1}(Q)
\end{array}
\end{aligned}
$$


where the horizontal sequences are portions of cohomology sequences, and the vertical mappings are induced by inclusion.

Let $L=\operatorname{Kern} g_{3} g_{2} g_{1}$. We must show that $g_{2} g_{1} L$ is f.g.

The group $h_{1} i_{3} L$ is f.g., since $X$ has $(P, Q, \sim)^{r+1}$, and consequently $i_{2} g_{1} L$ is f.g. Denote the kernel of the mapping $i_{2}$ of $g_{1} L$ by $K$. We can represent $g_{1} L$ as a direct sum $K+H$, where $H \approx i_{2} g_{1} L$ and is f.g. Also, $K$ has antecedent $K^{\prime}$ in $h^{r}(P \cap M)$.

Now $\delta\left(\phi_{3} \phi_{2} K^{\prime}\right)=g_{3} g_{2} \delta_{2} K^{\prime}=g_{3} g_{2} K \subset g_{3} g_{2} g_{1} L=0$. Hence the group $\phi_{3} \phi_{2} K^{\prime}$ has antecedent $K_{2}$ in $h^{r}(X)$, and $K_{2}$ is f.g., since $X$ has $(X, Q)^{r}$. Hence $j K_{2}=\phi_{3} \phi_{2} K^{\prime}$ is f.g., and $K^{\prime}$ may be represented as a direct sum $K_{0}+H_{0}$, where $K_{0}=\operatorname{Kern} \phi_{3} \phi_{2}$ and $H_{0}$ is f.g. Evidently $K_{0}$ is based on cobounding cocycles of $M$ and since $M$ has property $(P, Q, \sim)^{r}$, $\phi_{2} K_{0}$ must be f.g. It follows that $\delta_{1} \phi_{2} K^{\prime}=\delta_{1} \phi_{2} K_{0}+\delta_{1} \phi_{2} H_{0}$ is f.g., and therefore that $g_{2} \delta_{2} K^{\prime}=g_{2} K$ is f.g. Finally, then, $g_{2} g_{1} L=g_{2} K+g_{2} H$ is f.g.

Theorem II.2b. If $X$ has property $(P, Q)^{r}$ and $M$ is a closed subset of $X$ such that $X-M$ has property $(P, Q, \sim)^{r+1}$, then $M$ has property $(P, Q, \sim)^{r}$.

Proof. Again let $R, Q$ be a canonical pair of open subsets of $X$, and let $P$ be an open set with compact closure such that $R \supset P \supset Q$. Consider the following diagram:

$$
\begin{aligned}
& h^{r}(M) \stackrel{\delta_{1}}{\longrightarrow} h^{r+1}(X-M) \\
& \begin{array}{c}
h^{r}(R) \stackrel{j_{1}}{\rightarrow} h^{r}(R \cap M) \\
\uparrow i \\
h^{r}(P) \stackrel{\phi_{2}}{\rightarrow} h^{r}(P \cap M) \stackrel{\phi_{3}}{\cap} \stackrel{\delta_{2}}{\rightarrow} h^{r+1}(P-M)
\end{array} \mid i_{2} \\
& \begin{array}{c}
\uparrow \phi_{1} \\
h^{r}(Q \cap M) \stackrel{\delta_{3}}{\rightarrow} h^{r+1}(Q-M) .
\end{array}
\end{aligned}
$$

Let $L=\operatorname{Kern} \phi_{3} \phi_{2} \phi_{1}$. We must show that $\phi_{2} \phi_{1} L$ is f.g.

Since $i_{2} i_{1}\left(\delta_{3} L\right)=\delta_{1} \phi_{3} \phi_{2} \phi_{1} L=0, \delta_{3} L$ must be based on cobounding cocycles of $X-M$; and since $X-M$ has $(P, Q, \sim)^{r+1}, i_{1}\left(\delta_{3} L\right)$ must be f.g. It follows, by commutativity, that $\delta_{2} \phi_{1} L$ is f.g. Denote the kernel of the mapping $\delta_{2}$ of $\phi_{1} L$ by $K ; K$ is a subgroup of $h^{r}(P \cap M)$ and has an antecedent $K^{\prime}$ in $h^{r}(P)$.

The group $i K^{\prime}$ is f.g., since $X$ has $(P, Q)^{r}$, and therefore $j_{1} i K^{\prime}$ $=\phi_{2} j_{2} K^{\prime}=\phi_{2} K$ is f.g. Now $\phi_{1} L$ is representable as a direct sum $K+H$, where $H \approx \delta_{2}\left(\phi_{1} L\right)$, and $\phi_{2} \phi_{1} L=\phi_{2} K+\phi_{2} H$. It follows that $\phi_{2} \phi_{1} L$ is f.g., since both $\phi_{2} K$ and $H$ are. 
It is to be expected that the " $(P, Q)$ " and " $(P, Q, \sim)$ " properties are related. These relations are exhibited in the following two lemmas:

Lemma II.1. In order that a subset $M$ of $X$ have property $(P, Q)_{r}$, it is necessary and sufficient that $p_{r}(M) \leqq \omega$ and that $M$ have property $(P, Q, \sim)_{r}$. In particular, if $M$ is compact, then " $p_{r}(M) \leqq \omega$ " may be replaced by " $p_{r}(M)$ be finite."

PROOF OF NECESSITY. In the sequence of homomorphisms

$$
h_{r}(M \cap Q) \stackrel{i_{1}}{\rightarrow} h_{r}(M \cap P) \stackrel{i_{2}}{\rightarrow} h_{r}(M)
$$

induced by inclusion ( $P, Q$ being any canonical pair), $\operatorname{Im} i_{1}$ is f.g., so that a fortiori $\operatorname{Im} i_{2} i_{1}$ is f.g., implying that $p_{r}(M) \leqq \omega$. That $(P, Q)_{r}$ implies $(P, Q, \sim)_{r}$ is trivial.

Proof of SUfFICIENCY. Referring again to the sequence of the preceding paragraph, we are given that $\operatorname{Im} i_{2} i_{1}$ is f.g. since $p_{r}(M) \leqq \omega$. Let Kern $i_{2} i_{1}=K$; then $h_{r}(M \cap Q)$ may be represented as a direct sum $K+G$ where $G \approx \operatorname{Im} i_{2} i_{1}$. Evidently $K$ is generated by bounding cycles of $M$, so that $i_{1} K$ is f.g. since $M$ has property $(P, Q, \sim)_{r}$. Consequently $\operatorname{Im} i_{1}$ is f.g.

Lemma II.2. If $M$ is a closed or open subset of a locally compact space $X$, then in order that $M$ have property $(P, Q)^{r}$ it is necessary and sufficient that $p^{r}(M) \leqq \omega$ and $M$ have property $(P, Q, \sim)^{r}$. In particular, if $M$ is compact, then " $p^{r}(M) \leqq \omega$ " may be replaced by " $p^{r}(M)$ be finite."

By virtue of Lemma II.2, we may combine Theorems II.2a and II. $2 \mathrm{~b}$ to give:

Theorem II.2 (Second Fundamental DUality theorem). Let $X$ be a locally compact space having properties $(P, Q)^{r}$ and $(P, Q, \sim)^{r+1}$, and let $M$ be a closed subset of $X$. Then a necessary and sufficient condition that $M$ have property $(P, Q, \sim)^{r}$ is that $X-M$ have property $(P, Q, \sim)^{r+1}$.

Remark. Theorem II.2 replaces Theorem XI.3.4 of [13, p. 328], which assumed not only that $X$ is compact, but that $X$ had only the property $(P, Q, \sim)^{r}$ instead of $(P, Q)^{r}$. That the latter requirement was insufficient is shown by the conversion of the set $A \cup B$ of Example I.1 into a locally connected space $X$ by the addition of intervals from lines parallel to the $x$-axis as follows: $L_{1}=\{(x, 0) \mid 0 \leqq x \leqq 1 / \pi\}$; $L_{2}$ is a segment from the line $y=1 / 2$ and $L_{3}$ a segment from the line $y=-1 / 2$; and so on (see figure). The resulting space $X$ has property $(P, Q, \sim)_{0}$ since $X$ is locally connected, and property $(P, Q, \sim)_{1}$ 


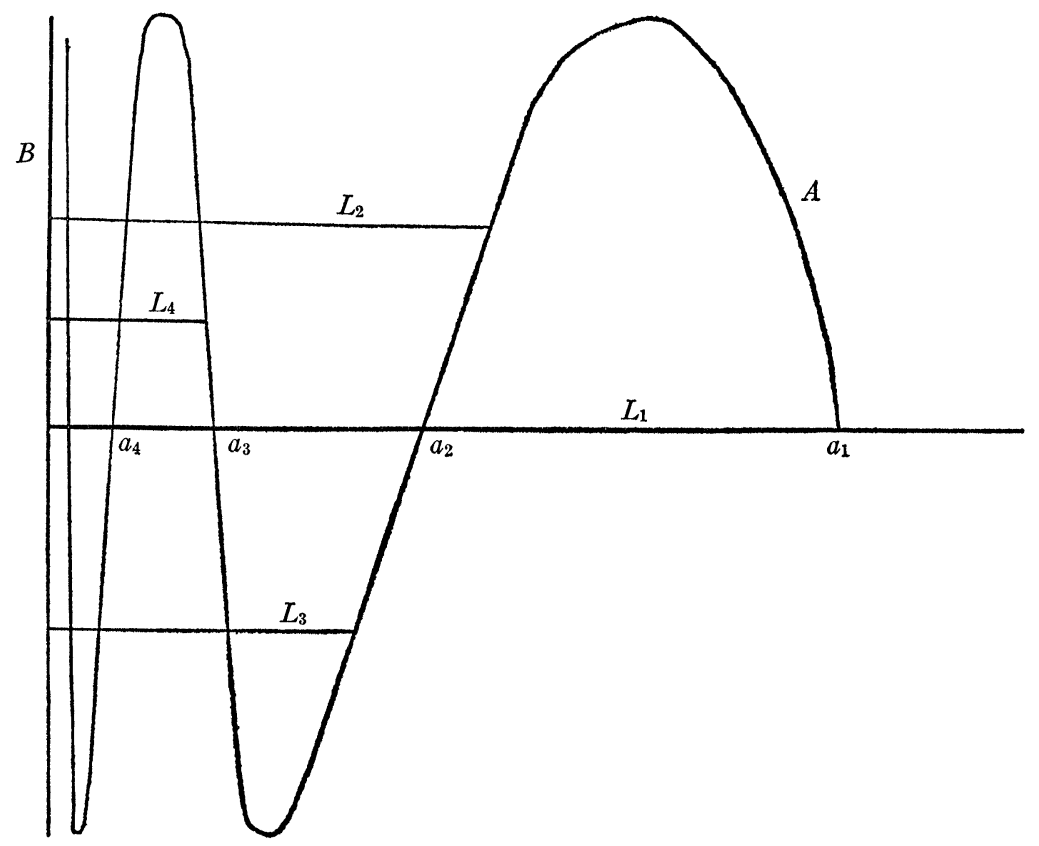

trivially. Hence by Theorem II.1, $X$ has properties $(P, Q, \sim)^{1}$ and $(P, Q, \sim)^{2}$.

Now let $M$ be a closed subset of the space $X$ (of the preceding paragraph) forming a configuration homeomorphic to the set $A \cup B$ of Example II.1 but consisting only of the points of Example II.1 having non-negative coordinates and of alternating segments $a_{1} a_{2}$, $a_{3} a_{4}, \cdots$, of $L_{1}$. Then $M$ does not have property $(P, Q, \sim)_{0}$ since it is not locally connected; and hence $M$ does not have property $(P, Q, \sim)^{1}$ by Theorem II.1. But $X-M$ does have property $(P, Q, \sim)^{2}$ trivially. Note, incidentally, that $p^{1}(X)=\infty$, so that $X$ cannot have property $(P, Q)^{1}$ and the hypothesis of Theorem II.2b is not satisfied for $r=1$. Incidentally, it will be noted that the proof of the sufficiency of Theorem XI.3.4 of $\left[13\right.$, p. 328] actually assumed property $(P, Q)^{r}$, and the proof of the necessity thereof assumed that $p^{r}(S)$ was finite.

For our purposes below, we recall the following relations (proved in [17] by methods extendible immediately to the case of the compact cohomology groups): With $M \subset X$,

(i) $p^{r}(M) \leqq p^{r}(X)+p^{r+1}(X-M)$,

(ii) $p^{r}(X) \leqq p^{r}(M)+p^{r}(X-M)$,

(iii) $p^{r+1}(X-M) \leqq p^{r}(M)+p^{r+1}(X)$. 
TheOREM II.3a. Let $M$ be a closed subset of $X$, and suppose that $p^{r}(X) \leqq \omega$ and $X$ has property $(P, Q)^{r+1}$. If $M$ has property $(P, Q)^{r}$, then $X-M$ has property $(P, Q)^{r+1}$.

Proof. By Lemma II.2, $p^{r}(M) \leqq \omega$, and $p^{r+1}(X) \leqq \omega$. Hence by relation (iii) above, $p^{r+1}(X-M) \leqq \omega$. By Theorem II.2a, $X-M$ has property $(P, Q, \sim)^{r+1}$. It now follows from Lemma II.2 that $X-M$ has property $(P, Q)^{r+1}$.

TheOREM II.3b. Let $M$ be a closed subset of $X$ and suppose that $X$ has property $(P, Q)^{r}$. If $X-M$ has property $(P, Q)^{r+1}$, then $M$ has property $(P, Q)^{r}$.

Proof. By Lemma II.2, $p^{r+1}(X-M) \leqq \omega$, and $p^{r}(X) \leqq \omega$. It follows from relation (i) that $p^{r}(M) \leqq \omega$. By Theorem II.2b, $M$ has property $(P, Q, \sim)^{r}$. It now follows from Lemma II.2 that $M$ has property $(P, Q)^{r}$.

From the last two theorems we have:

Theorem II.3 (Alternative Form of SeCond FUndamental DUALITY THEOREM). Let $M$ be a closed subset of $X$ and suppose that $X$ has properties $(P, Q)^{r}$ and $(P, Q)^{r+1}$. Then a necessary and sufficient condition that $M$ have property $(P, Q)^{r}$ is that $X-M$ have property $(P, Q)^{r+1}$.

REMARK. Theorem II.3 may, of course, be proved directly and independently of Theorems II.2a and II.2b.

We next prove a Poincaré-like type of duality for the " $P, Q$ " properties. For the definition of an orientable $n$-dimensional generalized manifold (abbreviated, orientable $n$-gm; compact $n$-gm is denoted by $n$-gcm), we refer to $[13$, VIII; 16].

Theorem II.4 (Third Fundamental DUality theorem). Let $U$ be an open subset of an orientable $n$-gm $M$. Then for $U$ to have property $(P, Q, \sim)^{r}, 1<r \leqq n$, it is necessary and sufficient that $U$ have property $(P, Q, \sim)_{n-r}$.

Proof. Consider the following diagram:

$$
\begin{gathered}
h^{r}(M) \stackrel{\alpha_{1}}{\rightarrow} h_{n-r}(M) \\
\uparrow \phi_{2} \\
h^{r}(P \cap U) \stackrel{\alpha_{2}}{\rightarrow} h_{n-r}(P \cap U) \\
\uparrow \phi_{1} \\
h^{r}(Q \cap U) \stackrel{\alpha_{3}}{\rightarrow} h_{n-r}\left(Q \cap \psi_{1}\right.
\end{gathered}
$$


The sets $P, Q$ form a canonical pair, and the $\phi$ 's and $\psi$ 's are homomorphisms induced by inclusions; while the $\alpha$ 's are isomorphisms onto by virtue of the Poincaré type of duality between the compact homology and compact cohomology groups of open subsets of an orientable $n$-gm (see [13, p. 260, 5.16]).

Let $L=\operatorname{Kern} \phi_{2} \phi_{1}$ and $K=\operatorname{Kern} \psi_{2} \psi_{1}$. Since $\alpha_{1} \phi_{2} \phi_{1} L=\psi_{2} \psi_{1} \alpha_{3} L=0$, it is clear that $\alpha_{3} L \subset K$; and by symmetry, $\alpha_{3}^{-1} K \subset L$, so that $\alpha_{3} L=K$.

To show that $\phi_{1} L$ is f.g. in case $\mathrm{U}$ has property $(P, Q, \sim)_{n-r}$, note that $\phi_{1} L=\alpha_{2}^{-1} \psi_{1} \alpha_{3} L=\alpha_{2}^{-1} \psi_{1} K$, and that $\psi_{1} K$ is f.g. since $U$ has property $(P, Q, \sim)_{n-r}$.

Similarly, if $U$ has property $(P, Q, \sim)^{r}, \phi_{1} L$ is f.g. and consequently $\psi_{1} K=\alpha_{2} \phi_{1} \alpha_{3}^{-1} K=\alpha_{2} \phi_{1} L$ is f.g.

REMARK. Theorem II.4 is a strengthening of Theorem XI.3.5 of [13, p. 329], inasmuch as in the latter theorem it was assumed that $M$ was compact as well as acyclic in dimensions $r-1$ and $r-2$.

We leave to the reader the proof of the corresponding form of duality for the general " $P, Q$ " properties:

Theorem II.4a (Alternative Form of third Fundamental DUALITY THEOREM). Let $U$ be an open subset of an orientable $n$-gm $M$. Then for $U$ to have property $(P, Q)^{r}$ it is necessary and sufficient that $U$ have property $(P, Q)_{n-r}$.

From the above duality theorems, one easily derives the following theorem:

Theorem II.5 (Fourth Fundamental DUality theorem). If $F$ is a closed subset of an orientable $n$-gm $M$, then a necessary and sufficient condition that $F$ have property $(P, Q, \sim)_{r}$ for an $r$ such that $0 \leqq r<n-1$, is that $M-F$ have property $(P, Q, \sim)_{n-r-2}$.

For the proof, we recall that every generalized manifold has the " $P, Q$ " properties in all dimensions, both in homology and cohomology (see $[13$, p. $244,1.1]$ ), so that Theorem II.2 applies; the proof is then obtained from the succession of equivalences of Theorems II.1, II.2 and II.4.

REMARK. Theorem II.5 is a strengthening of Theorem XI.1.1 of $[13,316]$, in which it was assumed that $M$ was compact as well as acyclic in dimensions $r, r+1$ and $r+2$.

The set $M$ of Example I.2, which we noted has property $(P, Q, \sim)_{1}$, has a complement $E^{3}-M$ having property $(P, Q, \sim)_{0}$-an instance of the above duality. However, $M$ does not have property $(P, Q)_{1}$.

It is also interesting to note the application of Theorem II.5 to the positional properties of Peano continua in euclidean spaces. This 
is the case $r=0$, where the local connectedness of a continuum becomes equivalent to the property $(P, Q, \sim)_{0}$. Thus Theorem II.5 shows that in any euclidean $n$-space (or orientable $n$-manifold), the Peano continua are positionally characterized by their complements having property $(P, Q, \sim)_{n-2}$.

It is instructive to observe why the complement of the configuration $A \cup B$ of Example I.1, imbedded in $E^{2}$ or $E^{3}$, fails to have prop$\operatorname{erty}(P, Q, \sim)_{n-2}$.

For the general " $P, Q$ " properties, the following duality holds:

THEOREM II.6. If $F$ is a closed subset of an orientable $n$-gm $M$, then $a$ necessary and sufficient condition that $F$ have property $(P, Q)^{r}$, for an $r$ such that $1 \leqq r \leqq n-1$, is that $M-F$ have property $(P, Q)_{n-r-1}$.

Proof. By Theorem II.3, for $F$ to have property $(P, Q)^{r}$ is equivalent to $M-F$ having property $(P, Q)^{r+1}$, and the latter, by Theorem II.4a, is equivalent to $M-F$ having property $(P, Q)_{n-r-1}$.

REMARK. Theorem II.6 is analogous to the well-known Alexander Duality Theorem; note, however, that it is not necessary to impose any acyclicity conditions on the manifold as in the case of the Alexander theorem. Incidentally, as originally proved in $[13$, p. 330 , Theorem 3.12], acyclicity in dimensions $r-1, r$ and $r+1$ was required, as was also compactness of the manifold.

LEMMA II.3. If $F$ is a closed subset of an $n$-gm $M$, then $p^{r}(F) \leqq \omega$ if and only if $p^{r+1}(M-F) \leqq \omega$. In particular, $p^{r}(F)$ is finite if and only if $p^{r+1}(M-F)$ is finite.

Lemma II.3 follows from the relations (i) and (iii) preceding Theorem II.3a (with $M, F$ replacing $X, M$ respectively), and the fact that $p^{r}(M) \leqq \omega$ for all $r$.

LEMMA II.4. If $F$ is a closed subset of an $n$-gm $M$, then $p_{r}(F) \leqq \omega$ if and only if $p_{r+1}(M, F) \leqq \omega$. In particular, $p_{r}(F)$ is finite if and only if $p_{r+1}(M, F)$ is finite.

Proof. Relations (i)-(iii) may be duplicated for compact homology, with $h_{r}(X, M)$ replacing $h^{r}(X-M)$. The relations analogous to (i) and (iii), with $M, F$ replacing $X, M$ respectively, are

(i') $p_{r}(F) \leqq p_{r}(M)+p_{r+1}(M, F)$,

(iii') $p_{r+1}(M, F) \leqq p_{r}(F)+p_{r+1}(M)$.

THEOREM II.7. If $F$ is a closed subset of an orientable $n$-gm $M$, then $a$ necessary and sufficient condition that $F$ have property $(P, Q)_{r}$, for an $r$ such that $0 \leqq r<n-1$, is that $M-F$ have property $(P, Q, \sim)_{n-r-2}$ and that $p_{r+1}(M, F) \leqq \omega$. 
Proof. By Lemma II.1, for $F$ to have property $(P, Q)_{r}$ is equivalent to $p_{r}(F) \leqq \omega$ and $F$ having property $(P, Q, \sim)_{r}$. By Theorem II.5, for $F$ to have property $(P, Q, \sim)_{r}$ is equivalent to $M-F$ having property $(P, Q, \sim)_{n-r-2}$. And by Lemma II.4, $p_{r}(F) \leqq \omega$ is equivalent to $p_{r+1}(M, F) \leqq \omega$.

REMARK. Theorem II.7 generalizes Theorem XI.1.4 of [13, p. 319]; in the latter theorem, compactness of $M$ as well as acyclicity in dimensions $r, r+1$ and $r+2$ were assumed. When $M$ is compact, the condition $p_{r+1}(M, F) \leqq \omega$ may be replaced by $p_{n-r-1}(M-F)$ finite.

ExAmple II.1. In cartesian 3-space, $E^{3}$, let $X$ denote the subspace obtained by combining the set $B$ of Example I.1 in the $(x, y)$-plane with a hollow tube $T$ replacing $A$ and converging to $B$ in the same manner as $A$ (a picture of this configuration may be found in [13, p. 337]). Although $p_{2}\left(E^{3}, X\right)=0, E^{3}-X$ fails to have property $(P, Q, \sim)_{0}$ and hence $X$ fails to have property $(P, Q)_{1}$.

In Example I.2, $M$ has property $(P, Q, \sim)_{1}$, but not $(P, Q)_{1}$; $X-M$ has property $(P, Q, \sim)_{0}$, but $p_{2}(X-M)=\infty$.

THEOREM II.8. If $F$ is a closed subset of an orientable $n$-gm $M$, and both $p_{r}(F)$ and $p^{r+1}(F)$ are $\leqq \omega$, then for $F$ to have property $(P, Q)_{r}$, where $0 \leqq r<n-1$, it is necessary and sufficient that $M-F$ have property $(P, Q)_{n-r-2}$.

Proof of NECESSITy. By Theorem II.7, $M-F$ has property $(P, Q, \sim)_{n-r-2}$. By Lemma II.3, $p^{r+2}(M-F) \leqq \omega$, implying (by duality) $p_{n-r-2}(M-F) \leqq \omega$. That $M-F$ has property $(P, Q)_{n-r-2}$ now follows from Lemma II.1.

Proof of sufficiency. By Lemma II.4, $p_{r+1}(M, F) \leqq \omega$. That $F$ has property $(P, Q)_{r}$ now follows from Theorem II.7.

Remark. Compare Theorem XI.1.6 of [13, p. 320].

III. Relations with local connectedness. It is well known that if a compact space is $l^{n}$ at every point, then the $1 c^{n}$ property of the space is expressible globally as in the chain-realization lemma of Lefschetz [4]. The principal motive for the Lefschetz lemma was to establish the complex-like character of the compact $1 \mathrm{c}^{n}$ spaces, so far as their homology groups of dimensions up to and including $n$ are concerned; more precisely, to prove the existence of a certain complex $K$ such that the homology groups $H^{r}(X)$, for all $r \leqq n$, of the compact $\mathrm{lc}^{n}$ space $X$, are isomorphic with subgroups of the groups $H^{r}(K)$ of corresponding dimensions. When the coefficient group is a field, so that the homology groups become vector spaces over the field, then the dimensions themselves-the Betti numbers-are finite and suffice to characterize the groups; and in this case $K$ can be so 
selected that the isomorphisms are onto.

The medial properties that we have defined enable us to throw new light on and to generalize these results. That there exists a relationship between " $P, Q$ " properties and "lc" properties is evident from the easily proved fact that for any $r$, if $X$ has property $(P, Q)_{r}$, then it is $r$-lc. That the converse fails is evident from the following example:

EXAMPLE III.1. In the cartesian plane let

$$
\begin{aligned}
& X_{1}=\{(x, y) \mid 0 \leqq x \leqq 1, y=0\}, \\
& X_{2}=\{(x, y) \mid x=0,0 \leqq y \leqq 1\}, \\
& X_{3}=\{(x, y) \mid 0 \leqq x \leqq 1, y=1\},
\end{aligned}
$$

and $L_{n}=\{(x, y) \mid x=1 / n, 0 \leqq y \leqq 1\}, n=1,2,3, \cdots ;$ and $X=\mathrm{U}_{i=1}^{3} X_{i}$ $\cup \cup_{n=1}^{\infty} L_{n}$. Then $X$ is 1 -lc but does not have property $(P, Q)_{1}$.

However, as we have remarked above, for $r=0$ the equivalence does hold; and, more generally, the property of $X$ being $l^{n}$ and the possession of $(P, Q)_{r}$ for $r=0,1, \cdots, n$ are equivalent. A much more revealing, as well as more general, result, is embodied in the following theorem:

TheOREM III.1. If a locally compact space $X$ has property $(P, Q, \sim)_{n}$ and is $(n+1)$-lc, then $X$ has property $(P, Q)_{n+1}$.

REMARK. In view of the comments above, this theorem could be stated as an equivalence: For locally compact spaces having property $(P, Q, \sim)_{n}$, the local property $(n+1)$-lc and the medial property $(P, Q)_{n+1}$ are equivalent.

Proof of Theorem III.1. Since $X$ is locally compact, to show that it has property $(P, Q)_{r}$ is equivalent to showing that for every canonical pair $P, Q$ of open sets, $H_{r}(\bar{Q} \mid \bar{P})$ is f.g. And if $P^{\prime}, Q^{\prime}$ form another canonical pair such that $P \supset P^{\prime} \supset Q^{\prime} \supset Q$, then for $H_{r}\left(\bar{Q}^{\prime} \mid \bar{P}^{\prime}\right)$ to be f.g. implies $H_{r}(\bar{Q} \mid \bar{P})$ f.g., as is shown by the sequence

$$
H_{r}(\bar{Q}) \rightarrow H_{r}\left(\bar{Q}^{\prime}\right) \rightarrow H_{r}\left(\bar{P}^{\prime}\right) \rightarrow H_{r}(\bar{P})
$$

of homomorphisms induced by inclusions.

Now since $X$ is $(n+1)$-lc, there exists for each $x \in X$ and open set $U$ containing $x$ a canonical pair $P(x), Q(x)$ of open sets containing $x$ and contained in $U$ such that $H_{n+1}(\bar{Q}(x) \mid \bar{P}(x))=0$. If $Q^{\prime}(x)$ is an open set such that $x \in Q^{\prime}(x) \subset Q(x)$, then $P(x), Q^{\prime}(x)$ will be called a special canonical pair of neighborhoods for $x$.

Suppose $P, Q$ a given canonical pair of open subsets of $X$. Then there exists a finite set of special canonical pairs of neighborhoods $P\left(x_{i}\right), Q^{\prime}\left(x_{i}\right)$ such that if $Q_{k}^{\prime}=\cup_{i=1}^{k} Q^{\prime}\left(x_{i}\right), P_{k}=\bigcup_{i=1}^{k} P\left(x_{i}\right)$, then 
$Q \subset \bar{Q}_{k}^{\prime} \subset \bar{P}_{k} \subset P$, and as remarked above, we need only show that $H_{n+1}\left(\bar{Q}_{k}^{\prime} \mid \bar{P}_{k}\right)$ is f.g. If $k=1$, this is trivial, so that we may use induction on $k$; precisely, by showing that if $H_{n+1}\left(\bar{Q}_{k}^{\prime} \mid \bar{P}_{k}\right)$ is f.g. for all choices of $P, Q$, etc., and $k \leqq m$, then $H_{n+1}\left(\bar{Q}_{m+1}^{\prime} \mid \bar{P}_{m+1}\right)$ will be f.g.

Consider the following diagram:

$$
\begin{aligned}
& H_{n+1}\left(\bar{P}_{1}\right)+H_{n+1}\left(\bar{P}_{2}\right) \stackrel{\phi_{1}}{\longrightarrow} H_{n+1}(\bar{P}) \\
& H_{n+1}\left(\bar{R}_{1}\right)+H_{n+1}\left(\bar{R}_{2}\right) \stackrel{\phi_{2}}{\longrightarrow} H_{n+1}(\bar{R}) \stackrel{\Delta_{2}}{\longrightarrow} H_{n}\left(\bar{R}_{1} \cap \bar{R}_{2}\right) \\
& \uparrow g_{1} \uparrow h \\
& H_{n+1}(\bar{Q}) \stackrel{\Delta_{3}}{\longrightarrow} H_{n}\left(\bar{Q}_{1} \cap \bar{Q}_{2}\right)
\end{aligned}
$$

where (1) the horizontal rows are portions of Mayer-Vietoris sequences [3, p. 39], (2) the vertical homomorphisms are induced by inclusions, and (3) $\bar{P}_{1}, \bar{P}_{2}$ and $\bar{P}$ represent, respectively, unions of $m \bar{P}\left(x_{i}\right)$ 's, a single $\bar{P}\left(x_{m+1}\right)$ and $\bar{P}_{1} \cup \bar{P}_{2} ;(4)$ the $\bar{R}$ 's and $\bar{Q}$ 's are obtained from the corresponding special canonical pairs $P\left(x_{i}\right), Q^{\prime}\left(x_{i}\right)$ with a new $R\left(x_{i}\right)$ such that $P\left(x_{i}\right) \supset Q\left(x_{i}\right) \supset R\left(x_{i}\right) \supset Q^{\prime}\left(x_{i}\right)$; the $P\left(x_{i}\right)$, $R\left(x_{i}\right)$ are special canonical pairs, so that the induction assumption applies to them; and $Q=Q_{m}^{\prime} \cup Q^{\prime}\left(x_{m+1}\right)$ etc.

We must show that $g_{2} g_{1} H_{n+1}(\bar{Q})$ is f.g.

Let $B_{0}=\operatorname{Im} \phi_{2}$. Then, since $\operatorname{Im} \phi_{1} k_{2}$ is f.g. by the induction assumption and the $(n+1)$-lc hypothesis, $g_{2} B_{0}=\operatorname{Im} \phi_{1} k_{2}$ is also f.g.

Since $X$ has property $(P, Q, \sim)_{n}, h\left(\operatorname{Im} \Delta_{3}\right)$ is f.g. It follows that $\Delta_{2}\left(\operatorname{Im~} g_{1}\right)$ is f.g., and hence that $\operatorname{Im} g_{1}$ is representable as a direct sum $B_{0}^{\prime}+N$ where $B_{0}^{\prime} \subset B_{0}$ and $N$ is f.g. Hence $g_{2} g_{1} H_{n+1}(\bar{Q})$ is f.g. I

In considering a property that is defined relative to a special dimension, it is frequently useful to extend the property over a range of dimensions. For instance, except for the initial dimension 0 , the $n$-lc property does not as a rule impose enough restriction upon a space to obtain fruitful results. It is for this reason that so much attention has been centered on the "lc ${ }^{n}$ " spaces, i.e., spaces that are $r$-lc for $r=0,1, \cdots, n$. We shall find, similarly, that the " $P, Q$ " properties are frequently more useful when extended to more than one dimension. In the case of local connectedness, we let the symbol $l_{k}^{n}$ signify property $r$-lc for $r=k, k+1, \cdots, n$, although instead of " $c_{0}^{n}$ " we continue to use the conventional "lc $n$ ". For the " $P, Q$ " properties we define:

Definition III.1. A set will be said to have $\operatorname{property~}_{k}(P, Q)_{n}, k \leqq n$, if it has property $(P, Q)_{r}$ for $r=k, k+1, \cdots, n$; although instead of ${ }_{n}(P, Q)_{n}$ we continue to use the symbol $(P, Q)_{n}$. The symbol 
${ }_{k}(P, Q, \sim)_{n}$ is defined similarly; and likewise ${ }^{k}(P, Q)^{n},{ }^{k}(P, Q, \sim)^{n}$ for cohomology.

Since property $(P, Q)_{r}$ implies $r$-lc, one obtains from Theorem III.1 by induction:

THEOREM III.2. If a locally compact space $X$ has property $(P, Q, \sim)_{k}$ for some non-negative integer $k$ and is $\operatorname{lc}_{k+1}^{n}$ for some $n>k$, then $X$ has property $_{k+1}(P, Q)_{n}$.

Corollary III.1. Under the same hypothesis as in Theorem III.2, $p_{r}(X) \leqq \omega$ for $r=k+1, k+2, \cdots, n$; and if $X$ is compact, then these numbers are finite.

CoROLLARY III.2. Under the same hypothesis as in Theorem III.2, there exists a locally finite complex $K$ such that $p_{r}(K)=0$ for $r \leqq k$ and $p_{r}(K)=p_{r}(X)$ for $r=k+1, k+2, \cdots, n$; if $X$ is compact, $K$ may be taken as a finite complex.

CoRollary III.3. For locally compact spaces having property $(P, Q)_{k}$, the properties $\mathbf{l c}_{\mathbf{k}}^{n}$ and ${ }_{k}(P, Q)_{n}$ are equivalent.

Theorem III.3. For every compact space $X$, the following properties I, II and III are equivalent:

I. $X$ has property $(P, Q)_{k}$, is $\mathbf{l c}_{\boldsymbol{k}+1}^{n}$, and $p_{n+1}(X)$ is finite.

II. $X$ has property ${ }_{k}(P, Q)_{n}$, and $p_{n+1}(X)$ is finite.

III. $X$ has property ${ }^{k+1}(P, Q)^{n+1}$ and $p_{k}(X)$ is finite.

Proof. That I implies II follows from Theorem III.2; and that II implies I follows from the fact that $(P, Q)_{r}$ implies $r$-lc. That properties II and III are equivalent may be shown as follows: By Lemma II.1, II is equivalent to property ${ }_{k}(P, Q, \sim)_{n}$ and $p_{r}(X)$ finite for $r=k, k+1, \cdots, n+1$. By Lemma II.2, III is equivalent to ${ }^{k+1}(P, Q, \sim)^{n+1}$ and $p_{r}(X)$ finite for $r=k, k+1, \cdots, n+1$. By the first fundamental duality theorem, ${ }^{k+1}(P, Q, \sim)^{n+1}$ is equivalent to ${ }_{k}(P, Q, \sim)_{n}$.

IV. Positional properties; duality with lc properties. The results which we have been stating in \$III all have to do with medial properties " $P, Q$ " of the entire space. We return now to the kind of application exemplified in $\S I$ and II. For instance, in Theorem II.5 we established the duality between the 0-lc property of a subcontinuum of an orientable $n$-gm and the $(P, Q, \sim)_{n-2}$ property of its complement. Using the results established in III we can now give the analogous duality for general dimension $r$ and arbitrary closed subsets; for the theorems of §III translate lc properties into " $P, Q$ " properties and one can then use the " $P, Q$ " dualities. 
THEOREM IV.1. In order that a closed subset $F$ of an orientable $n$-gm $M$ should be $1_{k}^{r}$ and have property $(P, Q)_{k}$, where $k \leqq r \leqq n-2$, it is necessary and sufficient that $M-F$ have property ${ }_{n-r-2}(P, Q, \sim)_{n-k-2}$ and that $p_{s}(M, F)$ be $\leqq \omega$ for $s=k+1, k+2, \cdots, r+1$; when $F$ is compact, the conditions on the $p_{s}(M, F)$ are equivalent to each $p_{s}(M-F)$ being finite for $s=n-r-1, n-r, \cdots, n-k-1$.

Proof. By Corollary III.3, for $F$ to be $\mathrm{lc}_{\boldsymbol{k}}^{r}$ and have property $(P, Q)_{k}$ is equivalent to $F$ having property ${ }_{k}(P, Q)_{r}$; which, in turn, is equivalent, by Theorem II.7, to $M-F$ having the properties stated in the theorem. When $M$ is compact, $p_{s}(M, F)=p_{n-s}(M-F)$.

REMARK. When $k=0$, it is unnecessary to include the condition $(P, Q)_{k}$ since, as remarked before, 0 -lc and $(P, Q)_{0}$ are equivalent for locally compact sets; thus we have the important special case:

CoRollary IV.1. In order that a closed subset, $F$, of an orientable $n$-gm $M$ should be $1 \mathrm{c}^{r}$, where $r$ is a fixed integer such that $0 \leqq r \leqq n-2$, it is necessary and sufficient that $M-F$ have property ${ }_{n-r-2}(P, Q, \sim)_{n-2}$ and that $p_{s}(M, F)$ be $\leqq \omega$ for $s=1,2, \cdots, r+1$; when $M$ is compact, the latter condition may be replaced by the condition that the numbers $p_{s}(M-F)$ are finite for $s=n-r-1, n-r, \cdots, n-1$.

REMARK. Corollary IV.1 generalizes Theorem XI.2.1 of [13, p. 320] in which the manifold was assumed acyclic from dimensions 1 to $r+2$. Consider Example I.2; the set $M$ therein is 0 -lc, hence by Corollary IV.1, $E^{3}-M$ has property $(P, Q, \sim)_{1}$. The set $M$ has property $(P, Q, \sim)_{1}$, so that by Theorem II.5, $E^{3}-F$ has property $(P, Q, \sim)_{0}$. Also, $p_{2}\left(E^{3}-M\right)=0$ (we may consider $E^{3}$ compactified by a point at infinity). Hence the only part of the conclusion of Corollary IV.1 that is not satisfied is that $p_{1}\left(E^{3}-M\right)$ is not finite; and this accounts for the fact that $M$ is not 1-lc.

Another instructive example is the configuration shown on p. 340 of [13], with $S^{3}$ as the manifold in which it is imbedded; here the set $F$ is not 1-lc because $E^{3}-F$ fails to have property $(P, Q, \sim)_{0}$.

The characterization of $1 \mathrm{c}^{r}$ sets in an $n$-gm can be given in terms of the " $P, Q$ " properties of the complement alone. For this, we need the following lemma:

Lemma IV.1. If $U$ is an open subset of an $n$-gm $M$, then a necessary and sufficient condition that $U$ have property $(P, Q)_{n-1}$ is that $p_{n-1}(U)$ $\leqq \omega$.

Proof. This is an immediate consequence of Lemma II.1 and the following lemma: 
Lemma IV.1a. Every open subset $U$ of an $n$-gm has property $(P, Q, \sim)_{n-1}$.

Proof. Let $P, Q$ be any canonical pair. We may assume $U \neq M$, since every $n$-gm has property $(P, Q)_{n-1}$. Consider the following diagram:

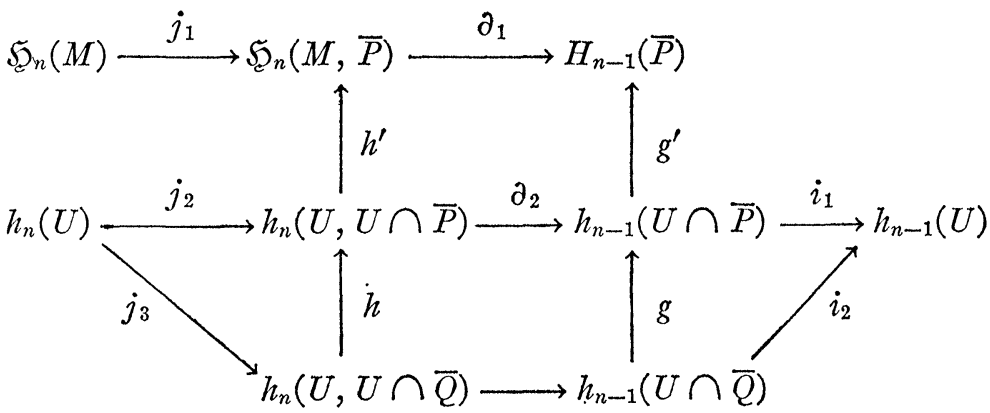

where the horizontal (and slant) sequences are homology sequences and the $g$ 's and $h$ 's are induced by inclusion.

Since $U \neq M, h_{n}(U)=0$ and hence $\partial_{2}$ and $\partial_{3}$ are isomorphisms into. Let $L=\operatorname{Kern} i_{2}$; then $\partial_{3} h_{n}(U, U \cap \bar{Q}) \approx L$. Evidently we wish to show that $g L$ is f.g. Suppose, however, that $g L$ is not f.g.; then Im $h$ is not f.g.

Now $h^{\prime}$ is an isomorphism into. For suppose $z \in h_{n}(U, U \cap \bar{P})$ such that $h^{\prime} z=0$. If $z_{n}$ is a representative cycle of $z$, this implies $z_{n}$ is in $U$, and $z_{n}=0 \bmod \bar{P}($ since $\operatorname{dim} M=n)$, and $z_{n}$ is in $\bar{P}$. That is, $z_{n}$ is in $U \cap \bar{P}$ and therefore $z=0$.

Consequently Im $h^{\prime} h$ is not f.g. However, $P, U \cap Q$ form a canonical pair and $M$, being a generalized manifold, has property $(P, Q)_{n-1}$, so that Im $g^{\prime} g$ is f.g. A fortiori, Im $\partial_{1} h^{\prime} h=\operatorname{Im} g^{\prime} g \partial_{3}$ is f.g. It follows that Kern $\partial_{1}$ is not f.g. But then $\operatorname{Im} j_{1}$ and hence $\mathfrak{S}_{n}(M)$ must not be f.g. in contradiction of the fact that $\operatorname{dim} \mathfrak{S}_{n}(M) \leqq 1$.

We can now state the following theorem (which generalizes Theorem XI.2.3 of $[13$, p. 321], in which the manifold was assumed to be acyclic in dimensions $1,2, \cdots, r+2)$ :

TheOREM IV.2. In order that a closed subset, $F$, of an orientable $n$-gcm $M$ should be $\mathrm{lc}^{r}$, where $r$ is a fixed non-negative integer $\leqq n-2$, it is necessary and sufficient that $M-F$ have properties ${ }_{n-r-1}(P, Q)_{n-1}$ and $(P, Q, \sim)_{n-r-2}$.

Proof. By Corollary IV.1, the lc $^{r}$ property of $F$ is equivalent to $M-F$ having property ${ }_{n-r-2}(P, Q, \sim)_{n-2}$ and $p_{s}(M-F)$ finite for $s=n-r-1, n-r, \cdots, n-1$. By Lemma II.1, these are in turn 
equivalent to $M-F$ having properties $(P, Q, \sim)_{n-r-2},{ }_{n-r-1}(P, Q)_{n-2}$ and $p_{n-1}(M-F)$ finite. By Lemma IV.1, $p_{n-1}(M-F)$ finite is equivalent to $M-F$ having property $(P, Q)_{n-1}$, so that the combined properties ${ }_{n-r-1}(P, Q)_{n-2}$ and $p_{n-1}(M-F)$ finite are equivalent to property ${ }_{n-r-1}(P, Q)_{n-1}$. |

In order to handle the $1 \mathrm{c}^{n-1}$ case, we need the following lemma (compare Lemma XI.2.4 of [13, p. 321]):

Lemma IV.2. If $F$ is a closed subset of an $n$-gm $M$ such that $p_{n-1}(F)$ $\leqq \omega$, then $F$ has property $(P, Q)_{n-1}$; and a fortiori, $F$ is $(n-1)$-lc.

Proof. By Lemma II.1, it is sufficient to show that $F$ has property $(P, Q, \sim)_{n-1}$; or, by virtue of the first fundamental duality, that $F$ has property $(P, Q, \sim)^{n}$. By the second fundamental duality, the latter is equivalent to $M-F$ having property $(P, Q, \sim)^{n+1}$, which it has, trivially, since $\operatorname{dim} M=n$.

REMARK. It is interesting to note that in the proof of Lemma IV.2, it is incidentally shown that every closed subset of an $n$-gm has property $(P, Q, \sim)^{n}$.

THEOREM IV.3. In order that a closed subset $F$ of an orientable $n$-gcm $M$ should be $1 \mathrm{c}^{n-1}$, it is necessary and sufficient that $M-F$ have prop$\operatorname{erty}_{0}(P, Q)_{n-1}$.

Proof of Necessity. Since $F$ is $1 \mathrm{c}^{n-2}, M-F$ has properties ${ }_{1}(P, Q)_{n-1}$ and $(P, Q, \sim)_{0}$ by Theorem IV.2. And since $F$ being $l^{n-1}$ implies $p_{n-1}(F)$ finite, and hence $p_{n}(M, F)$ finite by Lemma II.4, so must $p_{0}(M-F)$ be finite by duality. Thus $M-F$ has property $(P, Q)_{0}$ by Lemma II.1.

Proof OF SUfFiciency. Splitting the property $(P, Q)_{0}$ of $M-F$ into $(P, Q, \sim)_{0}$ and $p_{0}(M-F)$ finite, we apply Lemma IV.2 to show that $F$ is $(n-1)$-lc by virtue of the resulting finiteness of $p_{n-1}(F)$. That in addition $F$ is $l^{n-2}$ follows from Theorem IV.2.

Since the case where $F$ is a continuum is of special interest, the following theorem is noteworthy:

Theorem IV.4. In order that a subcontinuum $F$ of an orientable $n$-gcm $M$ be $\mathrm{lc}^{r}$, where $r \leqq n-2$, it is necessary and sufficient that $M-F$ have properties ${ }_{n-r-1}(P, Q)_{n-2}$ and $(P, Q, \sim)_{n-r-2}$.

Proof. Since $F$ is a continuum, $p_{n-1}(M-F)$ is finite and $M-F$ has property $(P, Q)_{n-1}$ by Lemma IV.1. Theorem IV.2 now applies.

CoROllary IV.2. In order that a separable subcontinuum $F$ of an orientable $n-\mathrm{gcm} M$ should be peanian, it is necessary and sufficient that $M-F$ have property $(P, Q, \sim)_{n-2}$. 
REMARK. For the case where $M$ is the 2 -sphere, the classical Schoenflies characterization of locally connected continua is a ready consequence of Corollary IV.2.

V. Duality between a domain and its boundary. The positional theorems in $\S I V$ have been concerned with the relations between a closed set $F$ and its entire complement $M-F$. However, it is of ten of special importance to know the relations between a single domain, $D$, in $n$-space and its boundary, or of the entire closed set to which it is complementary. One of the earliest theorems of this type was that of M. Torhorst (1921) [7]: If $F$ is a locally connected continuum in the euclidean plane, and $D$ is a domain complementary to $F$, then the boundary of $D$ is locally connected. That is, boundaries of domains complementary to Peano continua in the plane are themselves peanian.

In 1935, I generalized this theorem to $n$-space as follows [10]: If $F$ is a closed subset of $E^{n}$ which is $1 \mathrm{c}^{n-2}$, and $D$ is a domain complementary to $F$, then the boundary $F(D)$ of $D$ is 0 -lc; and in $[13$, p. 325, Theorem 2.19] I extended this to the framework of generalized manifolds, in that $F$ may be any $\mathrm{lc}^{n-2}$ closed subset of a spherelike $n$-gcm. Now since, as we saw in $\S I I I$, for locally compact spaces the properties $1 \mathrm{c}^{n-2}$ and ${ }_{0}(P, Q)_{n-2}$ are equivalent, both the Torhorst theorem and my generalization essentially assume the ${ }_{0}(P, Q)_{n-2}$ property of $F$. It appears, however, that by use of the " $P, Q$ " properties the conditions on $F$ can be considerably weakened. For this we need the following lemma, whose proof is elementary.

Lemma V.1. Let $X$ be a locally compact space and $U$ an open (resp. closed) subset of $X$ which is the union of disjoint open (resp. closed) sets $U_{0}$ and $U_{1}$. Then in order that $U$ have a given type of " $P, Q$ " property $\pi$, it is necessary and sufficient that $U_{0}$ and $U_{1}$ each have property $\pi$.

Theorem V.1 (Generalized Torhorst theorem). Let $M$ be an orientable $n$-gcm and $F$ a closed subset of $M$ which has properties $(P, Q)_{0}[$ or its equivalent $0-1 \mathrm{c}]$ and $(P, Q, \sim)_{n-2}$. Then if $D$ is a domain complementary to $F$, its boundary, $F(D)$ is 0-lc.

Proof. By Corollary IV.1, it is sufficient to show that $M-F(D)$ has property $(P, Q, \sim)_{n-2}$ and that $p_{n-1}(M-F(D))$ is finite.

Since $F$ is compact and 0 -lc, $p_{0}(F)$ is finite and consequently $p_{n-1}(M-F)$ is finite (cf. Lemma II.4). It follows that $p_{n-1}(D)$ is finite. Now $p_{n-1}(M-\bar{D}) \leqq p_{0}(\bar{D})+p_{n-1}(M)[17$, Theorem 2$]$, and as $\bar{D}$ is connected and $p_{n-1}(M)$ finite, it follows that $p_{n-1}(M-\bar{D})$ is finite. And since $p_{n-1}(M-F(D))=p_{n-1}(D)+p_{n-1}(M-\bar{D})$ by Lemma 
V.1, we conclude that $p_{n-1}(M-F(D))$ is finite.

Again, since $F$ is 0 -lc, $M-F$ has property $(P, Q, \sim)_{n-2}$ by Theorem IV.2. And since the sets $D, M-F-D$ are open, $D$ has property $(P, Q, \sim)_{n-2}$ by Lemma V.1. If, then, we can show that $M-\bar{D}$ has property $(P, Q, \sim)_{n-2}$, it will follow (again by Lemma V.1) that $M-F(D)$ has property $(P, Q, \sim)_{n-2}$. And to show that $M-\bar{D}$ has property $(P, Q, \sim)_{n-2}$, it is sufficient, by Corollary IV.1, to show that $\bar{D}$ is 0 -lc.

Suppose, then, that $\bar{D}$ is not 0 -lc. Then, since $\bar{D}$ is a continuum, there exist $[13$, p. 102, Theorem 2.1] open sets $P, R$ forming a canonical pair, and infinitely many components $D_{\nu}$ of $\bar{D} \cap(\bar{P}-R)$ that contain points of both $F(P)$ and $F(R)$. Let $P_{1}, R_{1}$ be a canonical pair of open sets such that $P \supset \bar{P}_{1}$ and $R_{1} \supset \bar{R}$. Since $D$ is dense in $\bar{D}$ and hence in the sets $D_{\nu}, h_{0}\left(D \cap\left(P_{1}-\bar{R}_{1}\right) \mid D \cap(P-\bar{R})\right)$ must be infinitely generated. But this is impossible since by hypothesis $F$ has property $(P, Q, \sim)_{n-2}$, so that by the Fourth Fundamental Duality Theorem, $M-F$ has property $(P, Q, \sim)_{0}$ and by Lemma V.1, $D$ has property $(P, Q, \sim)_{0}$; and $P-\bar{R}, P_{1}-\bar{R}_{1}$ form a canonical pair of open sets.

REMARK. When $n=2$, the $(P, Q, \sim)_{n-2}$ condition in the hypothesis of Theorem V.1 is of course already implied by the other condition.

Note, too, that if $p_{1}(M)=0$ and $F$ is a continuum, then $F(D)$ is a continuum ("Phragman-Brouwer property"; cf. [13, p. 242, Corollary $9.3]$ ); this would be the case if $M$ were the $n$-sphere, for example.

Corollary V.1. If $M$ is an orientable closed $n$-manifold in the classical sense such that $p_{1}(M)=0$, and $F$ is a Peano continuum in $M$ having property $(P, Q, \sim)_{n-2}$, then the boundary of every domain complementary to $F$ in $M$ is a Peano continuum.

The following theorem is in the nature of a dual to Theorem V.1:

TheOREM V.2. Let $M$ be an orientable $n$-gcm and $U$ an open subset of $M$ having properties $(P, Q, \sim)_{0}$ and $(P, Q, \sim)_{n-2}$ as well as $p_{n-1}(U)$ finite. Then the boundary of every component of $U$ is 0-lc.

Proof. Let $C$ be a component of $U$. Then $p_{n-1}(C)$ is finite. Also, $C$ has property $(P, Q, \sim)_{n-2}$ by Lemma V.1. Hence $M-C$ is 0 -lc by Corollary IV.1. And since $C$ has property $(P, Q, \sim)_{0}, M-C$ has property $(P, Q, \sim)_{n-2}$ by the Fourth Fundamental Duality Theorem. Then $F(C)$ is 0 -lc by Theorem V.1.

REMARK. Theorem V.2 is an " $n$-dimensional form" of the sufficiency part of the following theorem of R. L. Moore [5]: In order that the boundary of a simply connected domain, $D$, in $S^{2}$ should be 0-1c, it is necessary and sufficient that $D$ have property $S$. For property $S$ is here precisely property $(P, Q, \sim)_{0}\left(\right.$ and $\left.(P, Q, \sim)_{n-2}\right)$; while the 
simple connectedness of $D$ is equivalent to $p_{1}(D)=0$. (The necessity part of Moore's theorem is a corollary of Corollary IV.1 and Lemma V.1).

VI. $(X, Q)$-Properties, semi-r-connectedness, and relations to local $r$-connectedness. For a compact $\mathrm{lc}^{n}$ space to be complex-like in dimension $n+1$, it is not necessary that it be $(n+1)$-lc, since a weaker condition will suffice; this is the condition of semi- $(n+1)$-connectedness $[10 ; 13$, p. 167 , Definition 19.4]:

Definition VI.1. A space $X$ is called semi-r-connected at $x \in X$ if there exists a neighborhood $U$ of $x$ such that $h_{r}(U \mid X)=0$; and $X$ is called semi- $r$-connected if it is semi- $r$-connected at every point. Semi$r$-coconnectedness is defined analogously.

Analogous to Theorem III.1 we have:

Theorem VI.1. If a locally compact space $X$ has property $(P, Q, \sim)_{r}$ and is semi- $(r+1)$-connected, then it has property $(X, Q)_{r+1}$.

REMARK. That one cannot replace " $(X, Q)_{r+1}$ " in the conclusion of Theorem VI.1 by " $(P, Q)_{r+1}$ " as in Theorem III.1 is shown by the configuration of $[13, \mathrm{p} .340]$, which consists of a sequence of hollow cones, successively tangent along line elements, and converging to a line segment $L$. This configuration, $X$, as a subspace of $E^{3}$, is 0 -lc, hence has property $(P, Q, \sim)_{0}$; and $h_{1}(X)=0$ so that $X$ is semi-1connected. But $X$ cannot have property $(P, Q)_{1}$ since it is not 1-lc. However $X$ does have property $(X, Q)_{1}$ since $h_{1}(X)=0$.

The proof of Theorem VI.1 is similar to that of Theorem III.1, although simpler in that the top line of the diagram becomes the single term " $h_{n+1}(X)$."

Corollary VI.1. If $X$ has property $(P, Q, \sim)_{k}$ for some integer $k \geqq 0$, is $\mathrm{lc}_{k+1}^{n}$ for some $n>k$, and semi- $(n+1)$-connected, then $X$ has properties $_{k+1}(P, Q)_{n}$ and $(X, Q)_{n+1}$.

COROLlaRy VI.2. Under the hypothesis of Corollary VI.1, $p_{r}(X) \leqq \omega$ for $r=k+1, k+2, \cdots, n+1$. And if $X$ is compact, these numbers are all finite.

At this point we recall the analogue of Theorem III.1 for cohomology, since it admits of similar extensions:

THeOREM VI.2. If $X$ has property $(P, Q, \sim)^{r+1}$ and $p^{r}(x) \leqq \omega$ for all $x \in X$, then $X$ has property $(P, Q)^{r} .^{5}$

5 This appears as Theorem VI.7.2. of [13, p. 193], but with " $(P, Q)^{r+1}$ " instead of " $(P, Q, \sim)^{r+1}$ " in the hypothesis. Examination of the proof reveals, however, that the latter is sufficient. A proof by diagram may be given, of course, similar to that given above for Theorem III.1; see, for instance, [2, Proposition 6.2]. 
Corollary VI.3. For locally compact spaces having property $(P, Q, \sim)^{r+1}$, the property $(P, Q)^{r}$ is equivalent to $p^{r}(x) \leqq \omega$ at every point $x$.

To get the analogue of Theorem VI.1 for cohomology necessitates a better perspective on semi-r-coconnectedness. Consider the following definition:

Definition VI.2. Let $x \in X$ and $Q$ an open set containing $x$. Denote by $p^{r}(x ; Q, X)$ the dimension of $h^{r}(Q \mid X)$. Then for any open set $Q^{\prime}$ such that $x \in Q^{\prime} \subset Q, p^{r}\left(x ; Q^{\prime}, X\right) \leqq p^{r}(x ; Q, X)$. Let $p^{r}(x ; \infty)$ denote the greatest integer $k$, if it exists, such that if $U$ is an arbitrary open set containing $x$, there exists an open set $Q$ such that $x \in Q \subset U$ and $p^{r}(x ; Q, X)=k$. If no such integer exists, then we write $p^{r}(x ; \infty)$ $=\infty$. Note that semi- $r$-coconnectedness at $x$ corresponds precisely to the case where $p^{r}(x ; \infty)=0$.

Theorem VI.3. If $X$ has property $(P, Q, \sim)^{r+1}$ and $p^{r}(x ; \infty)$ is finite for all $x \in X$, then $X$ has property $(X, Q)^{r}$.

COROLlaRY VI.4. For locally compact spaces having property $(P, Q, \sim)^{r+1}$, property $(X, Q)^{r}$ is equivalent to $p^{r}(x ; \infty)$ being finite for every point $x$.

Corollary VI.5. If for some non-negative integer $k$, (1) $X$ has property $(P, Q, \sim)^{k+1},(2) p^{r}(x) \leqq \omega$ for all $x \in X$ and $r=m, m+1$, $\cdots, k$, where $0<m \leqq k,(3) p^{m-1}(x ; \infty)$ is finite for all $x \in X$, then $X$ has properties ${ }^{m}(P, Q)^{k}$ and $(X, Q)^{m-1}$.

In many theorems concerning compact spaces, replacement of an assumption of the finiteness of $p_{r}(X)$ by the assumption that $p_{r}(X)$ $\leqq \omega$, or its equivalent $(X, Q)_{r}$, allows of extension to the locally compact case. First let us note the following easily established lemmas:

Lemma VI.1. For locally compact spaces $X$, the following properties are successively weaker: (1) $p_{r}(X)$ finite, (2) $(X, Q)_{r},(3)$ semi-rconnectedness.

Lemma VI.2. For locally compact spaces $X$, the following properties are successively weaker: (1) $p^{r}(X)$ finite, (2) $(X, Q)^{r},(3) p^{r}(x, \infty)$ finite for all $x \in X$.

Theorem VI.4. In order that $X$ should have property $(P, Q)^{r}, 1 \leqq r$, it is necessary and sufficient that it have properties $(P, Q, \sim)_{r-1}$ and $(X, Q)$; similarly, $(P, Q)_{r}, 0 \leqq r$, is equivalent to the combined properties $(P, Q, \sim)^{r+1}$ and $(X, Q)_{r}$. 
Proof. By Lemma II.2, $(P, Q)^{r}$ is equivalent to properties $(P, Q, \sim)^{r}$ and $(X, Q)^{r}$. And by Theorem II.1, the latter properties are equivalent to $(P, Q, \sim)_{r-1}$ and $(X, Q)^{r}$.

Let us now inquire what relation exists between the properties $(X, Q)_{r}$ and $(X, Q)^{r}$ ?

Lemma VI.3. If $X$ has properties $(X, Q)_{r}$ and $(P, Q, \sim)_{r-1}$, then it has property $(X, Q)^{r}$.

Proof. Let $V$ be an open subset of $X$ with compact closure, and let $Q, U, W$ be open sets such that (1) $\bar{Q}$ is compact, (2) $Q \supset U \supset V$. We wish to show that $h^{r}(V \mid X)$ is f.g. This is equivalent to showing that in the sequence

$$
h^{r}(V) \stackrel{i_{1}}{\rightarrow} h^{r}(U) \stackrel{i_{2}}{\rightarrow} h^{r}(X),
$$

$\operatorname{Im} i_{2} i_{1}$ is f.g., or, alternatively, that in the dual sequence

$$
H_{r}(\bar{Q}, \bar{Q}-V) \stackrel{i_{1}^{*}}{\leftarrow} H_{r}(\bar{U}, \bar{U}-U) \stackrel{i_{2}^{*}}{\leftarrow} \mathfrak{S}_{r}(X),
$$

where $H_{r}(X, X-V)$ and $H_{r}(X, X-U)$ have been replaced by their equivalents (by excision) $H_{r}(\bar{Q}, \bar{Q}-V)$ and $H_{r}(\bar{U}, \bar{U}-U)$ respectively.

Consider the diagram

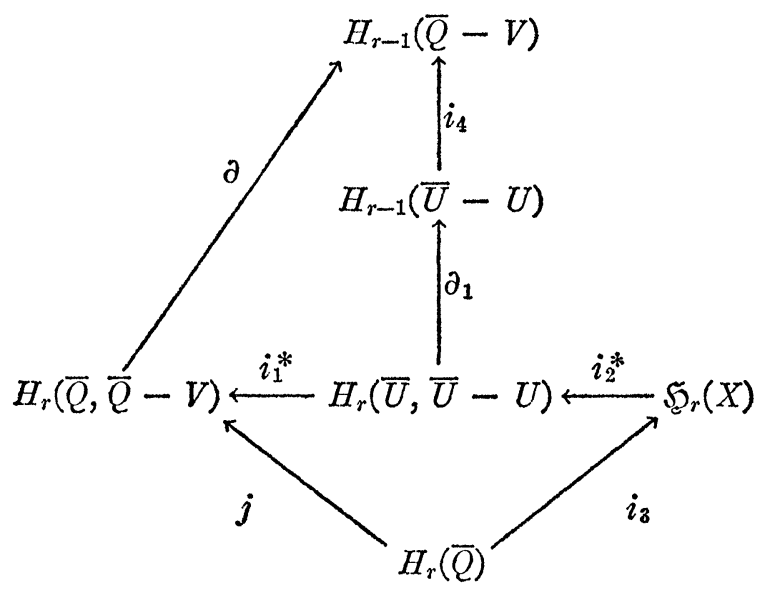

where $i_{3}$ and $i_{4}$ are induced by inclusion and the left-hand border is a portion of the exact homology sequence of the compact pair $\bar{Q}$, $\bar{Q}-V . \operatorname{Im} i_{3}$ is f.g. since $X$ has property $(X, Q)_{r}$ and hence $\operatorname{Im} i_{1}^{*} i_{2}^{*} i_{3}$ $=\operatorname{Im} j$ is f.g. Im $i_{4} \partial_{1}$ is f.g. since $X$ has property $(P, Q, \sim)_{r-1}$ and hence $\operatorname{Im} i_{4} \partial_{1} i_{2}^{*}=\operatorname{Im} \partial i_{1}^{*} i_{2}^{*}$ is f.g. Now Kern $\partial=\operatorname{Im} j$ is f.g., and therefore $\operatorname{Im} i_{1}^{*} i_{2}^{*}$ must be f.g., else $\operatorname{Im} \partial i_{1}^{*} i_{2}^{*}$ could not be f.g. 
Lemma VI.4. If $X$ has properties $(X, Q)^{r}$ and $(P, Q, \sim)^{r+1}$, then it has property $(X, Q)_{r}$.

Proof. With $V, Q, U$ as in the proof of Lemma VI.3, and $W$ an open set such that $\bar{U}-U \subset W \subset Q-\bar{V}$, we must show that in the sequence

$$
H_{r}(\bar{V}) \stackrel{i_{1}}{\rightarrow} H_{r}(\bar{U}) \stackrel{i_{2}}{\rightarrow} h_{r}(X),
$$

$\operatorname{Im} i_{2} i_{1}$ is f.g. Or, alternatively, that in the sequence

$$
H^{r}(\bar{V}) \stackrel{i_{1}^{*}}{\leftarrow} H^{r}(\bar{U}) \stackrel{i_{2}^{*}}{\leftarrow} \mathfrak{S}^{r}(X),
$$

in which we have replaced $H^{r}(X, X-V)$ by $H^{r}(\bar{V})$, etc., $\operatorname{Im} i_{1}^{*} i_{2}^{*}$ is f.g.

The proof follows from the following diagram by reasoning analogous to that employed in proving Lemma VI.3.

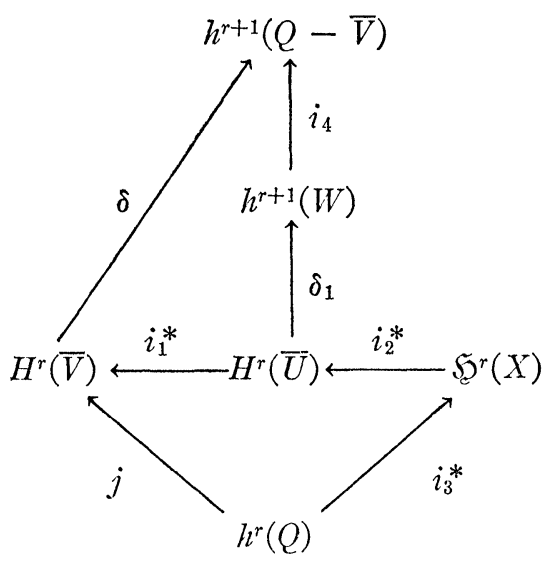

It will be noted that as a result of the implications stated in Lemmas VI.3 and VI.4, and Theorem VI.4, we have:

Lemma VI.5. For any locally compact space $X$, the combined properties $(P, Q)^{r}$ and $(X, Q)_{r-1}$ are equivalent to the combined properties $(P, Q)_{r-1}$ and $(X, Q)^{r}$.

THEOREM VI.5. If $X$ has property $(P, Q, \sim)_{r-1}, 1 \leqq r$, and is semi-rconnected, then it has property $(P, Q)^{r}$.

Proof. By Theorem VI.1, $X$ has property $(X, Q)_{r}$. Hence by Lemma VI.3, $X$ has property $(X, Q)^{r}$. The conclusion now follows from Theorem VI.4. 
REMARK. Theorem VI.5 is an extensive generalization of Corollary XI.3.8 of $\left[13\right.$, p. 330], to the effect that if a compact space is $1 c^{n}$, then it has property $(P, Q)^{n}$. Also it is of interest to compare Theorem VI.5 with Theorem III.1; the combined theorems imply that if a locally compact space $X$ has property $(P, Q, \sim)_{n}$ and is $(n+1)-\mathrm{lc}$, then $X$ has both property $(P, Q)_{n+1}$ and property $(P, Q)^{n+1}$.

Corollary VI.6. If $X$ has property $(P, Q, \sim)_{r-1}$ and is semi-rconnected, $1 \leqq r$, then $p^{r}(x) \leqq \omega$ for all $x \in X$.

REMARK. Corollary VI.6 is a generalization of Theorem VII.2.26 of $[13$, p. 211$]$ to the effect that if $X$ is $l^{n}$ and semi- $(n+1)$-connected, then $p^{n+1}(x) \leqq \omega$ for all $x \in X$. Of special interest in this connection is the fact that this leads to a generalization of a theorem stated in [14, Theorem 1] showing that if $X$ is $1 \mathrm{c}^{n}$, then it has no $n$-dimensional condensation; and if in addition $X$ is semi- $(n+1)$-connected, then it has no $(n+1)$-dimensional condensation. For in view of the Remark following Theorem VI.5, an $1 \mathrm{c}^{n}$ space has property $(P, Q)^{r}$ for all $r \leqq n$, while property $(P, Q)^{n}$ alone is sufficient to compel $p^{n}(x) \leqq \omega$ for all $x \in X$ and hence (see $[13$, p. 358, Corollary 1.12]) lack of $n$ dimensional condensation. Moreover, in view of Corollary VI.6 we can state:

Corollary VI.7. If $X$ has property $(P, Q, \sim)_{n}$ and is semi- $(n+1)$ connected, then $X$ has no $(n+1)$-dimensional condensation.

Theorem VI.6. If $X$ has property $(P, Q, \sim)^{r+1}$ and $p^{r}(x, \infty)$ is finite for all $x \in X$, then $X$ is $r$-lc and, moreover, has property $(P, Q)$.

Proof. By Theorem VI.3, $X$ has property $(X, Q)^{r}$. By Lemma VI.4, $X$ has property $(X, Q)_{r}$. And since, by Theorem II.1, property $(P, Q, \sim)^{r+1}$ is equivalent to $(P, Q, \sim)_{r}$, the latter property together with $(X, Q)_{r}$ gives property $(P, Q)_{r}$ by Lemma II.1. I

For the equivalences which follow, we shall use the following symbols: To denote that two properties $\pi_{1}$ and $\pi_{2}$ are equivalent, we write $\pi_{1} \Leftrightarrow \pi_{2}$; and to denote that properties $\pi_{1}$ and $\pi_{2}$ combined are equivalent to a property $\pi_{3}$, we write $\pi_{1}+\pi_{2} \Leftrightarrow \pi_{3}$. By $\pi_{1} \Rightarrow \pi_{2}$ we mean that $\pi_{1}$ implies $\pi_{2}$.

TheOREM VI.7. For every $X$ and $r \geqq 1$,

$$
(P, Q)^{r}+(P, Q, \sim)^{r+1} \Leftrightarrow(P, Q)_{r}+(P, Q, \sim)_{r-1} .
$$

Proof. By Lemmas II.1 and II.2 this is equivalent to proving that $(P, Q, \sim)^{r}+(X, Q)^{r}+(P, Q, \sim)^{r+1}$

$$
\Leftrightarrow(P, Q, \sim)_{r}+(X, Q)_{r}+(P, Q, \sim)_{r-1} .
$$


To show this, we recall that by Lemma VI.4,

$$
(P, Q, \sim)^{r+1}+(X, Q)^{r} \Rightarrow(X, Q)_{r}
$$

and that by the First Fundamental Duality Theorem,

$$
\begin{aligned}
& (P, Q, \sim)^{r+1} \Rightarrow(P, Q, \sim)_{r}, \\
& \quad(P, Q, \sim)^{r} \Rightarrow(P, Q, \sim)_{r-1} .
\end{aligned}
$$

Thus $\Rightarrow$ holds. To show that $\Leftarrow$ holds, we use the reverses of the last two relations above and the relation

$$
(P, Q, \sim)_{r-1}+(X, Q)_{r} \Rightarrow(X, Q)^{r}
$$

of Lemma VI.3.

Analogous to Theorem III.3, we can now state:

TheOREM VI.8. For every locally compact space $X$, the following properties I-III are equivalent, where $k<n$ :

I. $(P, Q)_{k}+\mathrm{lc}_{k+1}^{n}+(X, Q)^{n+1}$,

II. ${ }_{k}(P, Q)_{n}+(X, Q)^{n+1}$,

III. ${ }^{k+1}(P, Q)^{n+1}+(X, Q)_{k}$.

Proof. That I $\Leftrightarrow$ II follows as in Theorem III.3.

To show that $\mathrm{II} \Rightarrow \mathrm{III}$, we first note that $(P, Q)_{k} \Rightarrow(X, Q)_{k}$ trivially. Also, $r(P, Q)_{r+1} \Rightarrow(P, Q)^{r+1}$ for $r=k, k+1, \cdots, n-1$, by Theorem VI.7, so that ${ }_{k}(P, Q)_{n} \Rightarrow^{k+1}(P, Q)^{n}$. And finally, $(P, Q)_{n}+(X, Q)^{n+1}$ $\Rightarrow(P, Q)^{n+1}$ by Theorem VI.4.

That III $\Rightarrow$ II follows similarly from Theorems VI.4 and VI.7.

Theorem VI.9. If $X$ has property $(P, Q, \sim)^{k}$ and is $\operatorname{lc}_{k}^{n}, k \leqq n$, then $X$ has properties ${ }_{k}(P, Q)_{n}$ and ${ }^{k}(P, Q)^{n}$.

Proof. When $k=0$, the $(P, Q)_{0}$ and $(P, Q)^{0}$ properties follow from the 0 -lc and the ${ }_{1}(P, Q)_{n}$ and ${ }^{1}(P, Q)^{n}$ properties follow from the First Fundamental Duality Theorem and the Remark following Theorem VI.5. When $k>0$, the properties follow from the First Fundamental Duality Theorem and the Remark following Theorem VI.5.

THEOREM VI.10. If $X$ has property $(P, Q, \sim)^{n+1}$, then in order that $X$ have property $(P, Q, \sim)^{k}$ and be $\mathrm{lc}_{k}^{n}, k \leqq n$, it is necessary and sufficient that $p^{r}(x) \leqq \omega$ for all $x \in X$ and $r=k, k+1, \cdots, n$.

The necessity follows from Theorem VI.9, since property $(P, Q)^{r}$ implies $p^{r}(x) \leqq \omega$ for all $x$.

To prove the sufficiency: By Theorem VI.2, $X$ has property ${ }^{k}(P, Q)^{n}$, and a fortiori ${ }^{k}(X, Q)^{n}$; and since $X$ also has property 
${ }^{k+1}(P, Q, \sim)^{n+1}, p^{r}(x, \infty)$ is finite for all $x \in X$ by Corollary VI.4. Then by Theorem VI.6, $X$ is $\operatorname{lc}_{k}^{n}$.

COROLLARY VI.8. In order that an n-dimensional locally compact space $X$ should have property $(P, Q, \sim)^{k}$ and be $\operatorname{lc}_{k}^{n}, k \leqq n$, it is necessary and sufficient that $p^{r}(x) \leqq \omega$ for all $x \in X$ and $r=k, k+1, \cdots, n$.

TheOREM VI.11. For every $X$ the following equivalence holds: $(P, Q)_{r}$ $+\left[p^{r}(x) \leqq \omega\right.$ for all $\left.x \in X\right] \Leftrightarrow(P, Q)^{r}+r$-lc.

Proof. The case $r=0$ is trivial. For $r>0$, the $\Rightarrow$ is a consequence of Theorem VI.2 and the First Fundamental Duality Theorem, and the fact that $(P, Q)_{r} \Rightarrow r$-lc. The $\Leftarrow$ follows from Theorem III.1 and the First Fundamental Duality Theorem, and the fact that $(P, Q)^{r}$ $\Rightarrow p^{r}(x) \leqq \omega$ for all $x \in X$.

VII. Relations with uniform local connectedness. We recall (see $[13$, p. 292 , Definition 1.6]) that an open subset $U$ of a space $X$ is $r$-ulc (= uniformly locally connected in dimension $r$ ) if for arbitrary covering $\mathfrak{U}$ of $X$ (by open sets) there exists a refinement $\mathfrak{B}$ of $\mathfrak{U}$ such that if $Z_{r}$ is a compact cycle (augmented) of $U$ of diameter $<\mathfrak{B}$, then $Z_{r}$ bounds on a compact subset of $U$ of diameter $<\mathfrak{H}$. For open subsets of locally compact spaces, 0 -ulc and "ulc" (see [13, p. 109, Definition 4.7]) are equivalent, so that in such spaces, $r$-ulc is a generalization to arbitrary dimension of ulc.

It was shown in 1922 by R. L. Moore [5] that if an open subset $U$ of a compact space $X$ is ulc, then $U$ has "property $S$ " and hence property $(P, Q)_{0}$. In $[13]$ this result was generalized in the following manner: It was first shown that if an open subset $U$ of a regular space $X$ is $r$-ulc, then $q_{r}(U, x)=0$ for every $x \in X .^{6}$ It was then shown that if $U$ is a ulc ${ }^{k}$ open subset of an orientable $n$-gcm $M$, then $U$ has property ${ }_{0}(P, Q)_{k}{ }^{7}$ And later $[13$, p. 330 , Theorem 3.13$]$ it was shown that if an open subset $U$ of an orientable $n$-gcm $M$ (which is acyclic in dimensions $n-r-3$ to $n-r)$ has property $(P, Q)_{r}$, for some $r$ such that $0 \leqq r \leqq n-3$, and $q_{r+1}(U, x) \leqq \omega$ for all $x \in \bar{U}$, then $U$ has property $(P, Q)_{r+1}$. Both of these latter two results can be generalized to spaces that are not necessarily manifolds, as follows:

6 The numbers $q_{r}(U, x)$ correspond to the numbers " $q^{r}(U, x)$ " of [13, pp. 291-292]. For the result cited here, see [13, p. 293, Lemma 2.3].

${ }^{7}$ See $\left[13\right.$, p. 346, Theorem 6.8]. The symbols "ulc" ${ }^{k}$ " and "ulc ${ }_{k}^{n "}$ are defined analogously to the symbols " $\mathrm{c}^{k}$ " and " $\mathrm{lc}_{k}^{n}$ ". The converse of the result cited here does not generally hold, in that an open subset of an orientable $n$-gcm may have property ${ }_{0}(P, Q)_{k}$ yet not be ulck ${ }^{k}$; a simple example is the bounded domain $U$ in 3 -space whose boundary is the unit sphere $K$ together with the set of points interior to $K$ on the $x y$-plane for which $y>0$. 
THEOREM VII.1. If $U$ is an open subset of the locally compact space $X$, and $U$ has property $(P, Q, \sim)_{k}$ while $q_{k+1}(U, x) \leqq \omega$ for all $x \in \bar{U}$, then $U$ has property $(P, Q)_{k+1}$; and if $\bar{U}$ is compact, then $p_{k+1}(U)$ is finite.

Proof. For any canonical pair $P, Q$, we notice that each $x \in \bar{Q}$ lies in a pair of open sets $P^{\prime}, Q^{\prime}$ such that $P \supset P^{\prime} \supset \bar{Q}^{\prime}$ and such that $h_{k+1}\left(U \cap Q^{\prime} \mid U \cap P^{\prime}\right)$ is f.g. A finite union of such sets $Q^{\prime}$ covers $\bar{U} \cap \bar{Q}$ (since $\bar{Q}$ is compact), and the type of induction process employed in proving Theorems III.1 and VI.2 may be adapted to complete the proof.

CoRollary VII.1. If $U$ is an open subset of $X$ having property $(P, Q, \sim)_{k}$, then for $U$ to have property ${ }_{k+1}(P, Q)_{n}$ it is necessary and sufficient that $q_{r}(U, x) \leqq \omega$ for all $x \in \bar{U}$ and $r=k+1, k+2, \cdots, n$.

Proof. The sufficiency is a consequence of Theorem VII.1, using a step-wise process. The necessity results from the fact that the $(P, Q)_{r}$ property is stronger than the requirement of $q_{r}(x) \leqq \omega$, the latter being essentially a localization of the former.

The following corollary bears a strong analogy to Theorem III.2:

Corollary VII.2. If the open subset $U$ of $X$ has property $(P, Q)_{k}$ and is $\mathrm{ulc}_{k+1}^{n}, k<n$, then $U$ has property ${ }_{k}(P, Q)_{n}$.

Proof. As remarked above, for $U$ to be $r$-ulc implies that $q_{r}(U, x)$ $=0$ for all $x \in U$. We may then apply Corollary VII.1.

Since, if an open subset of a locally compact space is ulc, then it has property $(P, Q)_{0}$, we may therefore state:

Corollary VII.3. If an open subset $U$ of $X$ is ulc ${ }^{n}$, then $U$ has property $_{0}(P, Q)_{n}$.

However, although Corollary VII.3 provides the natural, obvious extension of the theorem of Moore cited, it will be noticed that even for $k=0$, Corollary VII.2 is actually stronger (since property $(P, Q)_{0}$ need not imply 0 -ulc).

Again using the method of proof indicated above for Theorem VII.1, we obtain:

THEOREM VII.2. If $U$ is an open subset of $X$, and $U$ has property $(P, Q, \sim)^{k+1}$ while $p^{k}(U, x) \leqq \omega$ for all $x \in \bar{U}$, then $U$ has property $(P, Q)^{k}$.

(The numbers $p^{k}(U, x)$ are defined in $[13$, p. 291], although as usual we here change the position of the dimensional index to conform to current usage.) 
Corollary VII.4. If $U$ is an open subset of an n-dimensional locally compact space such that $p^{r}(U, x) \leqq \omega$ for all $x \in \bar{U}$ and $r=k, k+1, \cdots, n$, then $U$ has property ${ }^{k}(P, Q)^{n}$.

VIII. Applications to continuous mappings. It is well-known that continuous mappings of compact locally connected spaces yield only locally connected spaces; and that the analogue holds for $(n-1)$ monotone mappings of $1 \mathrm{c}^{n}$ spaces, we showed in [15, Theorem 2]. Using " $P, Q$ " properties, these results may be substantially generalized.

First we recall that if $X$ and $X^{\prime}$ are locally compact spaces, then a mapping $f(X)=X^{\prime}$ is called proper if counter-images of compact subsets of $X^{\prime}$ are compact. Also, that $f$ is called $n$-monotone if all counter-images of points of $X^{\prime}$ are $r$-acyclic for $r=0,1, \cdots, n$.

Theorem VIII.1. Let $f(X)=X^{\prime}$ be a continuous, proper, $(n-1)$ monotone mapping of a locally compact space $X$ onto the locally compact space $X^{\prime}$. If $X$ has property $(P, Q)_{n}$, then $X^{\prime}$ has property $(P, Q)_{n}$.

Proof. See the proof of [15, Theorem 1]; or use left-hand portion of the diagram used in the proof of Lemma IX.1 below.

TheOREM VIII.2. Let $X$ have property $(P, Q, \sim)_{r}$ for some $r \geqq 0$, and be $(r+1)$-lc. If $f(X)=X^{\prime}$ is a continuous, proper, $r$-monotone mapping of $X$ onto the locally compact space $X^{\prime}$, then $X^{\prime}$ has property $(P, Q)_{r+1}$.

Proof. By Theorem III.1, $X$ has property $(P, Q)_{r+1}$. By Theorem VIII.1, $X^{\prime}$ has property $(P, Q)_{r+1}$.

CoROLlary VIII.1. Under the same hypothesis as Theorem VIII.2, $X^{\prime}$ is $(r+1)$-lc.

Corollary VIII.2. If $X$ has property $(P, Q, \sim)_{r}$, is $\mathrm{lc}_{r+1}^{\boldsymbol{k}}(r<k)$, and $f(X)=X^{\prime}$ is a continuous, proper, $(k-1)$-monotone mapping of $X$ onto the locally compact space $X^{\prime}$, then $X^{\prime}$ is $\mathrm{lc}_{r+1}^{k}$.

IX. Application to spatial decompositions. In [12] I studied decompositions of compacta into $\psi$-prime parts, the latter being the components of the closure, $\bar{S}$, of the set, $S$, of $\psi$-singular points and the points not in $\bar{S}$. A $\psi$-singular point is a point at which a given local topological property $\psi$ fails to hold. Of special interest is the property $\psi_{r}$ of being $r$-lc. A property $\psi$ is called expansive relative to a class $\Gamma$ of spaces if for $X \in \Gamma$, the failure of $X$ to have property $\psi$ at some point implies that the set $\bar{S}$ of $X$ has nondegenerate components. In particular, the property $\psi_{n+1}$ is expansive relative to the class $C_{n}^{n+1}$ of compacta that are $\mathrm{lc}^{n}$ and have finite $(n+1)$-dimensional Betti numbers. 
As a consequence of results stated above, we may give the following generalization:

THEOREM IX.1. The property $\psi_{n+1}$ of being $(n+1)$-lc is expansive relative to the class $P_{n}^{n+1}$ of compact spaces that have property $(P, Q, \sim)_{n}$ and are semi- $(n+1)$-connected. Moreover, every $\psi_{n+1}$-singular point of an element $X$ of $P_{n}^{n+1}$ lies in a nondegenerate component of the closure $\bar{S}$ of the set $S$ of $\psi_{n+1}$-singular points of $X$.

Proof. Let $X \in P_{n}^{n+1}$ and $x \in X$ a point at which $X$ is not $(n+1)$ lc. By Theorem VII.2.24 of $[13$, p. 210], there exists an open set $U$ containing $x$ such that for every pair $V, W$ of open sets for which $x \in W \subset \bar{W} \subset V \subset \bar{V} \subset U, h_{n+1}(F(V) \mid U-\bar{W})$ is infinitely generated.

Suppose that the component of $\bar{S}$ determined by $x$ contains only $x$. Then $[13$, p. 100 , Theorem 1.2] there exists an open set $V$ containing $x$ such that $\bar{V} \subset U$ and such that $\bar{S} \cap F(V)=\varnothing$. And since $[(X-U) \cup \bar{S}] \cap F(V)=\varnothing$, there exists an open set $Q$ containing $F(V)$ such that $\bar{Q} \subset U-\bar{S}$. Let $U^{\prime}=V \cup Q$ and $W=V-\bar{Q}$. Then $F(V)$ is a compact subset of the locally compact subspace $U^{\prime}-\bar{W}$; and the latter space has property $(P, Q, \sim)_{n}$ and is $(n+1)$-lc. Hence by Theorem III.1, $U^{\prime}-\bar{W}$ has property $(P, Q)_{n+1}$. It follows that $h_{n+1}(F(V) \mid U-\bar{W})$ is f.g. But this is impossible because of the property of $U$ stated above.

For the next theorem, we need the following lemma:

Lemma IX.1. The property $(P, Q, \sim)_{n}$ is invariant under continuous, proper, $n$-monotone mappings of locally compact spaces; and hence by the First Duality, $(P, Q, \sim)^{n+1}$ is also invariant.

Proof. Let $f(X)=X^{\prime}$ be a continuous, proper, $n$-monotone mapping of the locally compact space $X$, and suppose $X$ has property $(P, Q, \sim)_{n}$. Let $P^{\prime}, Q^{\prime}$ be a canonical pair of open sets of $X^{\prime}$, and let $P=f^{-1}\left(P^{\prime}\right), Q=f^{-1}\left(Q^{\prime}\right)$. In the diagram

$$
\begin{array}{ccc}
h_{n}(Q) & \stackrel{i_{1}}{\rightarrow} h_{n}(P) \stackrel{i_{2}}{\rightarrow} h_{n}(X) \\
\downarrow f_{1} & \quad f_{2} \quad \downarrow f_{3} \\
h_{n}\left(Q^{\prime}\right) \stackrel{i_{1}^{\prime}}{\rightarrow} h_{n}\left(P^{\prime}\right) \stackrel{i_{2}^{\prime}}{\rightarrow} h_{n}\left(X^{\prime}\right)
\end{array}
$$

where the $i$ 's are induced by inclusion and the $f$ 's by the mapping $f$, the $f^{\prime}$ 's are isomorphisms. And if $K=\operatorname{Kern} i_{2} i_{1}$ and $L=i_{1} K$, then $L$ must be f.g. since $X$ has property $(P, Q, \sim)_{n}$. Hence if $K^{\prime}=\operatorname{Kern} i_{2}^{\prime} i_{1}^{\prime}$ and $L^{\prime}=i_{1}^{\prime} K^{\prime}, L^{\prime}$ must be f.g. 
Theorem IX.2. ${ }^{8}$ If $X \in P_{n}^{n+1}$ and the $\psi_{n+1}$-prime parts of $X$ are $r$-acyclic for $r=1,2, \cdots, n$, then the (quotient) space $X^{\prime}$ of $\psi_{n+1}$-prime parts of $X$ is $(n+1)$-lc.

Proof. By Theorem IX.1, $\psi_{n+1}$ is expansive relative to $P_{n}^{n+1}$. By Lemma IX.1, $X^{\prime}$ has property $(P, Q, \sim)_{n}$. By Corollary VI.2, $p^{n+1}(X)$ is finite and hence $p^{n+1}\left(X^{\prime}\right)$ is finite [1]. It follows that $X^{\prime}$ is semi- $(n+1)$-connected [13, p. 168 , Corollary 19.5], and $X^{\prime} \in P_{n}^{n+1}$. The theorem now follows from Theorem 1 of [12].

TheOREM IX.3. ${ }^{9}$ Let $k$ and $n$ be integers such that $-2<k<n$. Then the property $\psi_{k+1}^{n}$ of being $\mathrm{l}_{k+1}^{n}$ is expansive relative to the class $P_{\boldsymbol{k}}^{n}$ of compact spaces that have property $(P, Q, \sim)_{k}$ and finite $r$-dimensional Betti numbers for $r=k+1, \cdots, n$. And if $X \in P_{\mathbf{k}}^{n}$ and the $\psi_{k+1}^{n}$-prime parts of $X$ are $r$-acyclic for $r=1,2, \cdots, n-1$, then the (quotient) space of $\psi_{k+1}^{n}$-prime parts of $X$ is $1 \mathrm{c}_{k+1}^{n}$.

Proof. If $X$ were not $(k+1)$-lc, then by Theorem IX.1 it would have a nondegenerate $\psi_{k+1}$-prime part and a fortiori a nondegenerate $\psi_{k+1}^{n}$-prime part. Let $m$ be the largest of the numbers $k+1, k+2, \cdots$, $n$ such that $X$ is $r$-lc for $r=k+1, \cdots, m$ and suppose that $k+1<m$ $<n$ (the case $m=k+1$ having been disposed of). Then by Theorem III.2, $X$ has property $(P, Q, \sim)_{m}$, and by Theorem IX.2 it has a nondegenerate $\psi_{m+1}$-prime part and a fortiori a nondegenerate $\psi_{k+1^{-}}^{n}$ prime part.

If $X \in P_{k}^{n}$ and the natural map of $X$ into the space $X^{\prime}$ of $\psi_{k+1}^{n}$-prime parts of $X$ is $(n-1)$-monotone, then $X^{\prime} \in P_{k}^{n}$, as may be shown by methods similar to those used in proving Theorem IX.2. It follows [12] that $X^{\prime}$ is ${ }_{1} c_{k+1}^{n}$.

$\mathrm{X}$. Miscellanea. In this concluding section are listed some miscellaneous remarks. First, in our introductory comments, we remarked upon the historical connection between 0 -lc and $(P, Q)_{0}$, and later made use of the general equivalence of $\operatorname{lc}^{n}$ and ${ }_{0}(P, Q)_{n}$. Since the latter is by definition the property that $h_{r}(Q \mid P)$ is f.g. for every canonical pair $P, Q$ and $r=0,1, \cdots, n$, it follows by duality that in the mapping $H^{r}(X, X-P) \rightarrow{ }^{i} H^{r}(X, X-Q)$ induced by inclusion, $\operatorname{Im} i$ is f.g.; or, using the symbols of $[13$, p. 166] (with appropriate change in position of dimensional index) that the group $H^{r}(X: X, X-\bar{P} ; X, X-\bar{Q})$ is f.g. It is interesting to observe, however, that the analogous statement holds for homology. More generally, we have the following theorem:

${ }^{8}$ Compare Theorem 3 of [12].

${ }^{2}$ See Principal Theorem in [12]. 
Theorem X.1. If the locally compact space $X$ has property $(P, Q, \sim)^{k}$ and is $1 \mathrm{c}_{k}^{n}, k \leqq n$, then the groups $H_{r}(X: X, X-P ; X, X-Q), r=k$, $k+1, \cdots, n$ are f.g.; and if, in addition, $X$ is semi- $(n+1)$-connected, the same holds for $r=n+1$.

Proof. By Theorem VI.9, $X$ has property ${ }^{k}(P, Q)^{n}$, so that for every canonical pair $P, Q$, the groups $h^{r}(Q \mid P)$ are f.g. for $r=k$, $k+1, \cdots, n$. The first half of the conclusion of the theorem now follows by duality.

Again by Theorem VI.9, $X$ has property $(P, Q)_{n}$ and a fortiori $(P, Q, \sim)_{n}$; and if $X$ is semi- $(n+1)$-connected, then $X$ has property $(P, Q)^{n+1}$ by Theorem VI.5. The second half of the conclusion of the theorem now follows.

Secondly, we recall the original Vietoris theorem [8] to the effect that the group $H_{r}(X)$ of a compact metric space $X$ has a countable fundamental system of elements $Z_{r}^{i}$ such that each element of $H_{r}(X)$ is expressible in a natural manner (using the topology of $H_{r}(X)$ ) as an infinite polynomial in the $Z_{r}^{i}$ with coefficients in the basic field. This result was extended in [13, p. 189, Theorem 5.8] to all compact spaces that are imbeddable as $G_{\delta}$ 's in locally compact $1 \mathrm{c}^{n}$ spaces where $r \leqq n$. As a consequence of Theorem III.1 above, and using precisely the same arguments as in $[13$, p. 186, Theorem 5.1] and $[13$, p. 189, Theorem 5.8$]$, we have:

THeORem X.2. If the compact space $X$ is imbeddable as a $G_{\delta}$ in a locally compact space $S$ which has property $(P, Q, \sim)_{r-1}$ and is $r$-lc, $r>0$, then $H_{r}(X)$ has a countable fundamental system $\left\{Z_{r}^{i}\right\}$ of elements and $H^{r}(X)$ has a base $\left\{Z_{j}^{r}\right\}$ such that $Z_{r}^{i} \cdot Z_{j}^{r}=\delta_{b}^{j}$.

Thirdly, and finally, it was pointed out to me by Dr. John Gary that the proof of Theorem III.1 goes through just as well if it only be assumed that "sufficiently small" canonical pairs satisfy the $(P, Q, \sim)_{n}$ condition. In order to make this precise, we may proceed as follows:

Definition X.1. If $\mathbb{E}$ is a covering of $X$ by open sets, then a canonical pair of open sets $P, Q$, will be said to be of diameter $\angle E$ if there exists $E \in \mathbb{E}$ such that $P \subset E$.

Definition X.2. A space $X$ has a given " $P, Q$ " property $\pi$ restricted by a covering $\&$ if $X$ has property $\pi$ for every canonical pair $P, Q$ of diameter $<\&$. When $X$ has a given " $P, Q$ " property restricted by some covering $\&$, we may indicate this by saying that $X$ has the property in the small.

TheOREM X.3. The properties $n$-lc, $(P, Q)_{n}$ in the small, and $(P, Q)_{n}$ are successively stronger for locally compact spaces. 
Proof. That $(P, Q)_{n} \Rightarrow(P, Q)_{n}$ in the small $\Rightarrow n$-lc follows easily. That the converse of the first implication does not generally hold is shown by the space $X$ defined in Example III.1; for here $X$ has property $(P, Q)_{1}$ in the small but does not have property $(P, Q)_{1}$. That the converse of the second implication does not generally hold is shown by the configuration $M$ of $[13$, p. 237, 7.15], which is 1 -lc but does not have property $(P, Q)_{1}$ in the small.

The proof of Theorem III.1 goes through as before even if the assumption of " $(P, Q, \sim)_{n}$ " in the hypothesis is replaced by " $(P, Q, \sim)_{n}$ in the small," provided that the sets $P(x)$ are all taken as of diameter $<\&$, where $\mathbb{E}$ is a covering of $X$ such that $X$ has property $(P, Q, \sim)_{n}$ restricted by $\&$. It will also be found that other theorems above can be subjected to a similar modification, as for instance Theorems III.2, VI.1, VI.2 and VI.3.

\section{BIBLIOGRAPHY}

1. E. G. Begle, The Vietoris mapping theorem for bicompact spaces II, Michigan Math. J. vol. 3 (1955-56) pp. 179-180.

2. A. Borel, The Poincarê duality in generalized manifolds, Michigan Math. J. vol. 4 (1957) pp. 227-239.

3. S. Eilenberg and N. Steenrod, Foundations of algebraic topology, Princeton University Press, 1952. $1-18$.

4. S. Lefschetz, Chain-deformations in topology, Duke Math. J. vol. 1 (1935) pp.

5. R. L. Moore, Concerning connectedness im kleinen and a related property, Fund. Math. vol. 3 (1922) pp. 232-237.

6. W. Sierpinski, Sur une condition pour qu'un continu soit une courbe jordanienne, Fund. Math. vol. 1 (1920) pp. 44-60.

7. M. Torhorst, Über den Rand der einfach zusammenhängenden ebenen Gebiete, Math. Z. vol. 9 (1921) pp. 44-65.

8. L. Vietoris, Über den höheren Zusammenhang kompakter Räume und eine Klasse von zusammenhangstreuen Abbildungen, Math. Ann. vol. 97 (1927) pp. 454-472.

9. R. L. Wilder, Point sets in three and higher dimensions and their investigation by means of a unified analysis situs, Bull. Amer. Math. Soc. vol. 38 (1932) pp. 649-692.

10. - On locally connected spaces, Duke Math. J. vol. 1 (1935) pp. 543-555.

11. —- Property $S_{n}$, Amer. J. Math. vol. 61 (1939) pp. 823-832.

12. - Decompositions of compact metric spaces, Amer. J. Math. vol. 63 (1941) pp. 691-697.

13. — Topology of manifolds, Amer. Math. Soc. Colloquium Publications, vol. 32, 1949.

14. - Concerning a problem of Alexandroff, Michigan Math. J. vol. 3 (195556) pp. 181-185.

15. — Monotone mappings of manifolds, Pacific J. Math. vol. 7 (1957) pp. $1519-1528$.

16. —, Local orientability, Colloq. Math. vol. 6 (1958) pp. 79-93.

17. R. L. Wilder and J. P. Roth, On certain inequalities relating the Betti numbers of a manifold and its subsets, Proc. Nat. Acad. Sci. U.S.A. vol. 40 (1954) pp. 207-209.

UNIVERSITY OF Michigan 